\title{
Long Noncoding RNA RP11-357H14.17 Plays an Oncogene Role in Gastric Cancer by Activating ATF2 Signaling and Enhancing Treg Cells
}

\author{
Tang Xiaoli, ${ }^{1}$ Wang Wenting, ${ }^{1}$ Zhang Meixiang, ${ }^{2}$ Zuo Chunlei, ${ }^{1}$ and Hu Chengxia $\mathbb{D}^{3}$ \\ ${ }^{1}$ Department of Clinical Laboratory, The First People's Hospital of Lianyungang, Lianyungang, Jiangsu, China \\ ${ }^{2}$ Department of Clinical Laboratory, 2nd Affiliated Hospital of Nanjing Medical University, China \\ ${ }^{3}$ Department of Clinical Laboratory, Suqian Hospital of Traditional Chinese Medicine, Suqian, Jiangsu, China
}

Correspondence should be addressed to Hu Chengxia; huchengxia119@163.com

Received 20 November 2020; Revised 18 February 2021; Accepted 5 March 2021; Published 30 May 2021

Academic Editor: Somchai Chutipongtanate

Copyright (c) 2021 Tang Xiaoli et al. This is an open access article distributed under the Creative Commons Attribution License, which permits unrestricted use, distribution, and reproduction in any medium, provided the original work is properly cited.

\begin{abstract}
Background. Gastric cancer (GC) is one of the most common malignant tumors in the world. The potential functions and mechanisms of long noncoding RNAs (lncRNAs) in GC development are still unclear. It is of great significance to explore the prognostic value of LncRNA signatures for GC. Methods. LncRNAs differently expressed in GC and their prognostic value were studied based on The Cancer Genome Atlas (TCGA) database. The functional regulatory network and immune infiltration of RP11-357H14.17 were further studied using a variety of bioinformatics tools and databases. Results. We found that the high expression of RP11-357H14.17 was closely associated with shortened overall survival (OS) and poor prognosis in gastric cancer patients. We also found that its expression was related to clinical features including tumor volume, metastasis, and differentiation. Functional enrichment analysis revealed that RP11-357H14.17 is closely related to enhanced DNA replication and metabolism; ssGSEA analysis implied the oncogenic roles of RP11-357H14.17 was related to ATF2 signaling and Treg cell differentiation. Furthermore, we verified such link by using real-time PCR and IHC staining in human GC samples. Conclusion. We demonstrate that RP11-357H14.17 may play a crucial role in the occurrence, development, and malignant biological behavior of gastric cancer as a potential prognostic marker for gastric cancer.
\end{abstract}

\section{Introduction}

Gastric cancer (GC) is an invasive disease that continues to have a severe impact on global health [1]. Although its incidence has declined overall over the past few decades, GC is still ranked the fourth most common type of cancer and the second cancer-related deaths globally $[1,2]$. Although the GC incidence has declined because of improved nutrition, better food preservation, better prevention, and earlier diagnosis and treatment, the prognosis still remains poor [1]. GC is usually diagnosed at an advanced stage. Gastric cancer is a heterogeneous disease, requiring continuous attention and research in prevention, early detection, and new treatment options.
Long noncoding RNAs (lncRNAs) are a group of noncoding RNAs to be defined as more than $200 \mathrm{bp}$ in length with no protein-coding function [3]. LncRNAs were first discovered in 2002 in mice lacking of specific and complete open reading frames. After decades of researches, LncRNAs were proved to be an important transcriptional and translational regulator, involving in not only physiological but also pathological processes, including chromatin remodeling, transcription, posttranscriptional translation, cell proliferation, differentiation, and metabolic reprogramming. Abnormal expression of lncRNAs took part in the occurrence and development of a variety of malignancies, including prostate cancer [4], ovarian cancer [5], breast cancer [6], and gastric cancer [7]. The high expression of H19 [8], TUSC7 [9], 
MEG3 [10], and MALAT1 [11] is closely related to the poor prognosis of GC patients and promotes the proliferation, metastasis, and EMT of GC cells. These studies showed that lncRNA plays a crucial role in the prognosis and malignant biological behavior of GC.

In this study, real-time polymerase chain reaction (RTPCR) was used to evaluate the expression level of RP11357H14.17 in GC problems. We also assessed the relationship between RP11-357H14.17 levels and clinicopathological characteristics. Through bioinformatics analysis and experimental verification, its role in GC was predicted and analyzed. Our findings may contribute to a better understanding of the role of RP11-357H14.17 as a regulatory factor for GC and as a possible candidate target for new diagnosis and treatment.

\section{Materials and Methods}

2.1. Bioinformatics Analysis. The software R package limma (V.3.40.6) was used to calculate the fold-change of transcripts and to screen for differentially expressed genes (DEGs) in the RNA-seq cohort. A fold-change larger than two and an adjusted $p$ value less than 0.05 were set as the cut-off values for screening significant DEGs. Cluster analysis and heatmap generation were performed using the $\mathrm{R}$ package clusterProfiler (V.3.12.0) and pheatmap (V.1.0.12), respectively. 23 Gene Ontology (GO) and Kyoto Encyclopedia of Genes and Genomes (KEGG) pathway analyses for the dysregulated genes were performed by ClueGO25 (V.2.5.5), within Cytoscape (V.3.7.1). A false-discovery rate less than 0.05 was set as the cut-off value.

\subsection{The Prognosis of Dysregulated IncRNAs Analyzed with} GEPIA and Kaplan-Meier Plotter. GEPIA (http://gepia .cancer-pku.cn/) [12] is a newly web-based tool that contains sequencing expression data from 9736 tumor samples of 33 cancer types and 8587 normal samples. The database includes a variety of analysis modules such as differential gene expression analysis, survival and prognosis analysis, correlation analysis, and dimensionality reduction analysis. In this study, GEPIA database was employed to further analyze the expression and prognostic value of differentially expressed lncRNAs in STAD. The expression analysis of these genes performed by one-way ANOVA, and the filter criteria were as follows: $|\log 2 \mathrm{FC}|>1, p$ value $<0.05$, "median," hazards ratio (HR), and 95\% confidence interval. The Kaplan-Meier (KM) Plotter (http://kmplot.com) is an effective tool for detecting the prognosis of patients with tumors. According to the expression of lncRNAs, patients with EC were divided into two groups: high and low expression groups. The hazard ratio (HR) at a 95\% confidence interval and $\log$-rank $p$ values were also investigated online. The filter conditions were as follows: cancer: pan-cancer RNA-seq (Uterus corpus endometrial carcinoma); survival: overall survival (OS); follow-up threshold: 120 months.

2.2.1. The Cellular Localization of $\operatorname{lncRNAs.~UCSC~(https://~}$ genome-asia.ucsc.eduk/index.html) provides a web-based interface to help users browse the genetic information, view the genome annotation assembly, and download gene sequences. LNCipedia (https://lncipedia.org) is a free human lncRNA transcription sequence and structure annotation database. LncLocator (https://LncLocatorwww.csbio.sjtu.edu .cn/bioinf/lncLocator/) is based on integrated classifier to predict Incrna subcellular localization of free public platform. Only by using the sequence information of IncRNA, the distribution ratio of lncRNA in cytoplasm, nucleus, ribosome, cytoplasm, and exosome can be obtained rapidly. In this study, sequence information of IncRNAs was detected by UCSC and LNCipedia, and cellular localization of lncRNAs was determined by LncLocator.

2.2.2. Real-Time PCR. Total RNA in GC tissues was isolated using TRIzol reagent. The expression of genes of interest was detected using the syber-green-based real-time PCR. The primers for genes used in the study were listed in Table 1.

2.2.3. Patients. The hospital-based case-control study consists of 52 patients newly diagnosed with gastric cancer. All the subjects were recruited from the 1st People's Hospital of Lianyungang and Suqian Hospital of Chinese traditional medicine, between February 2016 and August 2019. Patients with other hematological disorders, previous history of cancers, radiotherapy, and chemotherapy were excluded. The cancer-free control subjects from the same geographic area showed no evidence of a genetic relationship with the cases. The patients were classified according to World Health Organization classification. This study was approved by the Institutional Review Board of the 1st People's Hospital of Lianyungang and Suqian Hospital of Chinese traditional medicine (LL-16-12 and SCY-17-15), and every patient had written informed consent. The clinical features of all the cases and controls were presented in Table 2.

2.2.4. IHC Staining. Sections were stained according to the previous publication. The section was incubated within primary mouse anti-human Ab for ATF2(ab239361), WT1(ab89901), CD4(ab183685), Foxp3(ab215206), and TGF- $\beta(\mathrm{ab} 215715)$; the sections were stained with $\mathrm{DAB}$ according to manufacturer's protocols and mounted and photographed using a digitalized microscope camera (Nikon, Japan).

\section{Results}

3.1. RP11-357H14.17 Is Overexpressed in Human Stomach Cancer. To fully screen the long noncoding RNA within human stomach cancer, we analyzed total 18036 lncRNAs within the Cancer Genome Atlas Stomach Adenocarcinoma (TCGA-STAD) data collection; we found 375 GC patients and 32 normal controls with the TCGA-STAD. All of the 18036 lncRNAs were compared within the GC patients and normal controls by using Limma $\mathrm{R}$ package; the results indicated that 3790 lncRNAs were significantly differently expressed (adjusted $p<0.05$ ) within these two groups, including 1642 down-regulated and 2148 up-regulated (Figure 1(a)). We then rearranged the up-regulated and down-regulated lncRNAs by $\log \mathrm{Fc}$ in decreasing order; we listed top 50 up- and down-regulated lncRNAs in Figures 1(b) and 1(c). Within these 100 lncRNAs, RP11$357 \mathrm{H} 14.17$ was the most up-regulated lncRNAs. 
TABLE 1: Primer sequence for genes of interest.

\begin{tabular}{|c|c|}
\hline Gene & Primer (forward/reverse) \\
\hline \multirow{2}{*}{ RP11-357H14.17 } & TCCGGCCATCTGGCGCT \\
\hline & CAAAGGCGACGTGCCGG \\
\hline \multirow{2}{*}{ RECQL4 } & GCGCTCTACCGGGAATACC \\
\hline & CAGCCCGATTCAGATGGGG \\
\hline \multirow{2}{*}{ ATAD5 } & GTGAAGGACTGCGAGATTGAG \\
\hline & TGTCTCTAGTCTTCCCTAGTGGT \\
\hline \multirow{2}{*}{ GINS1 } & ACGAGGATGGACTCAGACAAG \\
\hline & TGCAGCGTCGATTTCTTAACA \\
\hline \multirow{2}{*}{ ORC6 } & ACAAGGAGACATATCAGAGCTGT \\
\hline & AGTGGCCTGGATAAGTCAAGAT \\
\hline \multirow{2}{*}{ RAD51 } & ССТССТСТTTAACGССТССТG \\
\hline & GGGGACAACTCCCAGACTTTTT \\
\hline \multirow{2}{*}{ EME1 } & TCTGAGGAGTTGCCAACATTTG \\
\hline & GGCTTCACAATCTGAGATGTCAA \\
\hline \multirow{2}{*}{ CCND1 } & GCTGCGAAGTGGAAACCATC \\
\hline & ССТССТTCTGCACACATTTGAA \\
\hline \multirow{2}{*}{ DBF4 } & CCGGAAAGTCCTTTTACTTGGAT \\
\hline & AACССТCAATTACCCСАCССА \\
\hline \multirow{2}{*}{ CDC25C } & TCTACGGAACTCTTCTCATCCAC \\
\hline & TCCAGGAGCAGGTTTAACATTTT \\
\hline \multirow{2}{*}{ MCM2 } & ATGGCGGAATCATCGGAATCC \\
\hline & GGTGAGGGCATCAGTACGC \\
\hline \multirow{2}{*}{ NASP } & AGATTGGGAACCTAGAGCTTGC \\
\hline & ACTTCTCCGAGTTTAAGATGTGC \\
\hline \multirow{2}{*}{$\mathrm{EZH} 2$} & AATCAGAGTACATGCGACTGAGA \\
\hline & GCTGTATCCTTCGCTGTTTCC \\
\hline \multirow{2}{*}{ PCNA } & CCTGCTGGGATATTAGCTCCA \\
\hline & CAGCGGTAGGTGTCGAAGC \\
\hline \multirow{2}{*}{ CDC25A } & GTGAAGGCGCTATTTGGCG \\
\hline & TGGTTGCTCATAATCACTGCC \\
\hline \multirow{2}{*}{ KRT8 } & TGTGCCTACCTGCGGAAATC \\
\hline & CTATGACCGAGGTGTCTGAGA \\
\hline \multirow{2}{*}{ PARD6B } & TTGGAGCTGAATTTCGTCGGT \\
\hline & AGCCTACCAAAACGTCAACATT \\
\hline \multirow{2}{*}{ THOC6 } & TCCCAGAGCGTCTCACCAT \\
\hline & CCACCGGCTTCTTACTTTCCT \\
\hline \multirow{2}{*}{ WT1 } & ACCAAAAATCGGTTCTTCATCCC \\
\hline & TCACATCACTGGCAATCTTAGGA \\
\hline \multirow{2}{*}{ ATF2 } & CACCAGCAGCACGAGTCTC \\
\hline & TGTGCGAGGCAAACAGGAG \\
\hline \multirow{2}{*}{ FOXP3 } & GTGGCCCGGATGTGAGAAG \\
\hline & GGAGCCCTTGTCGGATGATG \\
\hline \multirow{2}{*}{ HAT1 } & AAGCCATTCGGAACCTTACTTC \\
\hline & AGTGCCATCTTTCATCATCCAC \\
\hline \multirow{2}{*}{ NUF2 } & GGAAGGCTTCTTACCATTCAGC \\
\hline & GACTTGTCCGTTTTGCTTTTGG \\
\hline \multirow{2}{*}{ PLK1 } & CAGTCACTCTCCGCGACAC \\
\hline & GAGTAGCCGAATTGCTGCTG \\
\hline
\end{tabular}

RP11-357H14.17 is located in chromesome 17 with a Ensembl ID of ENSG00000272763.1. We confirmed its expression in human GC tissues by using an online tool, Gepia [12]. By involving the samples from the GenotypeTissue Expression (GTEx) and TCGA-STAD (408 tumor samples and 211 normal control), we found that RP11$357 \mathrm{H} 14.17$ is overexpressed in human stomach cancer (Figure 1(d)). However, there is no significant difference in RP11-357H14.17 expression with different TMN stage (Figure 1(e)). The effect of RP11-357H14.17 on overall survival (OR) of GC patients was also analyzed which implied that high expression of RP11-357H14.17 was associated with poor prognosis of GC patients $(p=0.044)$ (Figure 1(f)). Furthermore, 52 paired human GC samples were collected for our study; we found that RP11-357H14.17 was overexpressed in GC tissues compared to paired adjacent tissues (Figure 1(g)). Moreover, we found that GC tissues with higher TMN stage was associated with high expression of RP11-357H14.17 (Figure 1(g)). The subcellular localization of RP11-357H14.17 was predicted by using an online tool: lncLocator; the result indicated that RP11-357H14.17 was mainly located in cytosol (36\%) and ribosome (30\%) (Figure 1(h)). The expression of RP11-357H14.17 was also significantly associated with poor histological differentiation (Table 2).

3.2. Increased Expression of RP11-357H14.17 Is Associated with Increased DNA Replication and Metabolism in Human Gastric Cancer. To further investigate the oncogenic roles of RP11-357H14.17 in human GC, first, we performed KEGG and GO enrichment analysis using up-regulated DEGs obtained by two comparison patterns. One is the comparison between RP11-357H14.17 top-32 expression GC samples and normal GC control samples ( $\mathrm{T}$ vs. N), and the other is the comparison between the GC samples with top 40 and bottom 40 RP11-357H14.17 expression (High40 vs. Low40). We figured out shared GO enrichment terms between two comparison described above including "DNA replication," "nuclear DNA replication" in Biological Processes (BP) GO terms, "mitochondrial protein complex," "respiratory chain" in the Cell Components (CC) GO terms, and "electron transfer activity" and "NADPH dehydrogenase activity" in Molecular Functions (MF) GO terms (Figures 2(a) and 2(b)). For KEGG enrichment, we found shared terms including "cell cycle" and "DNA replication" which implied similar events as GO enrichment (Figures 2(c) and 2(d)). These results might suggested that the overexpression of RP11357 H14.17 might seriously related to increased DNA replication and metabolism in human GC cells. In order to further confirm the shared terms implied by GO and KEGG enrichment, we selected a set of genes including "RECQL4, ATAD5, GINS1, ORC6, ARD51, EME1, and CCND1" for the GO term "DNA replication," and "HMGCR, DHFR, FASN, DECR1, DUS2, GRHPR, and MTRR" for the GO term "NADPH dehydrogenase activity" using 52 paired human GC samples. We found that these genes were highest in those RP11-357H14.17 higher expression GC samples, significantly lower in the RP11-357H14.17 lower expression GC samples, and lowest in adjacent normal tissues (Figures 2(e) and 2(f)). 
TABLE 2: Frequency distributions of selected variables in gastric cancer patients.

\begin{tabular}{|c|c|c|c|c|c|}
\hline \multirow{2}{*}{ Features } & \multicolumn{2}{|c|}{ RP11-357H14.17 ${ }^{\text {High }}$} & \multicolumn{2}{|c|}{ RP11-357H14.17 ${ }^{\text {High }}$} & \multirow[b]{2}{*}{$p$} \\
\hline & $N$ & $\%$ & $N$ & $\%$ & \\
\hline Age (years) & & & & & 0.779 \\
\hline$\leq 50$ & 10 & $19.23 \%$ & 12 & $23.08 \%$ & \\
\hline$>50$ & 16 & $30.77 \%$ & 14 & $26.92 \%$ & \\
\hline Gender & & & & & 0.999 \\
\hline Male & 14 & $26.92 \%$ & 13 & $25.00 \%$ & \\
\hline Female & 12 & $23.08 \%$ & 13 & $25.00 \%$ & \\
\hline Differentiation & & & & & 0.0254 \\
\hline G1-G2 & 17 & $32.69 \%$ & 8 & $15.38 \%$ & \\
\hline G3-G4 & 9 & $17.31 \%$ & 18 & $34.62 \%$ & \\
\hline Tumor size $(\mathrm{cm})$ & & & & & 0.0145 \\
\hline$\leq 5 \mathrm{~cm}$ & 16 & $30.77 \%$ & 7 & $13.46 \%$ & \\
\hline$>5 \mathrm{~cm}$ & 10 & $19.23 \%$ & 19 & $36.54 \%$ & \\
\hline TMN stage & & & & & 0.0118 \\
\hline I-II & 18 & $34.62 \%$ & 8 & $23.08 \%$ & \\
\hline III-IV & 8 & $15.38 \%$ & 18 & $26.92 \%$ & \\
\hline H. pylori infection & & & & & 0.999 \\
\hline Positive & 16 & $30.77 \%$ & 15 & $28.85 \%$ & \\
\hline Negative & 10 & $19.23 \%$ & 11 & $21.15 \%$ & \\
\hline
\end{tabular}

3.3. The Oncogenic Roles of RP11-357H14.17 Was Partially via Promoting ATF2 Signaling in Human Gastric Cancer. In order to further clarify the possible mechanism for RP11357H14.17-associated malignancy, we did single sample GSEA (ssGSEA) analysis using oncogenic signatures (c6) gene sets using "N vs. T" and "High 40 vs. Low40". We found that high expression of RP11-357H14.17 in stomach cancer tissues were associated with ATF2-related gene sets including "ATF2_s_UP v1_up," “ATF2_up. V1_up," and "ATF2_ up.V1_DN" (Figures 3(a) and 3(b)). We picked four genes including KRT8, PARD6B, THOC6, and WT1 which were involved in all three gene sets. Their expressions were confirmed in TCGA-STAD data indicating that RP11357H14.17 high expression samples have significantly higher expression of these four genes compared to RP11-357H14.17 low expression samples and normal control samples (Figures 3(c) and 3(d)). Next, we also investigated the expression of these four genes in 52 paired human GC samples; we found that all these four genes were significantly overexpressed in RP11-357H14.17 high expression samples and significantly lower in RP11-357H14.17 low expression samples and paired normal control samples (Figure 3(e)). Lastly, we analyzed the protein expression of ATF2 and WT1 in the 52 paired human GC samples; the result was consistent to the results obtained from real-time PCR; WT1 and ATF2 were significantly higher in RP11-357H14.17 high expression samples compared to RP11-357H14.17 low expression samples and paired normal control samples (Figure 3(f)).

3.4. RP11-357H14.17 Promotes GC Development by Inducing Treg-Associated Immunosuppression. To study the role of RP11-357H14.17 in tumor microenvironment (TME), we analyzed the immune-related roles of RP11-357H14.17 by
ssGSEA analysis using immunologic signatures (c7) including 4872 gene sets for two sample sets described above. The results from both comparisons indicated that RP11$357 \mathrm{H} 14.17$ was significantly associated with Treg cell percentage within human GC by sharing gene sets "GSE37532_TREG_VS_TCONV_CD4_TCELL_FROM_LN_ UP," “GSE24634_TREG_VS_TCONV_POST_DAY5_IL4_ CONVERSION_UP," “GSE18893_TCONV_VS_TREG_24 H_TNF_STIM_UP," “GSE14415_TCONV_VS_FOXP3_KO_ INDUCED_TREG_DN," and "GSE14415_NATURAL_TRE G_VS_TCONV_DN” (Figures 4(a) and 4(b)). Next, we have chosen one of the gene set "GSE37532_TREG_VS_TCONV_ CD4_TCELL_FROM_LN_UP” and grip its involving genes from the TCGA-STAD database. The results were presented in Figure 4(c); four genes including FOXP3, PLK1, and HAT1 NUF2 which all reported to be related to Treg cell differentiation were selected; their expression was analyzed in both the TCGA-STAD database and 52 paired human GC samples. We found the four Treg cell-related genes were highest in RP11-357H14.17 high expression GC samples, lower in RP11-357H14.17 low expression GC samples, and lowest in normal control samples (Figures $4(\mathrm{c})$ and $4(\mathrm{~d})$ ). Last, we detected Treg cells percentage as well as the immunosuppression effector, TGF- $\beta$ in human GC samples by using multiple-color IHC staining; we found that Treg cells were most abundant in RP11-357H14.17 high expression tissues, relatively lower in RP11-357H14.17 low expression tissues, and lowest in adjacently normal tissues (Figure 4(e)).

\section{Discussion}

LncRNA significantly regulates gene expression in both nucleus and cytoplasm [3]. In the nucleus, lncRNA binds to 


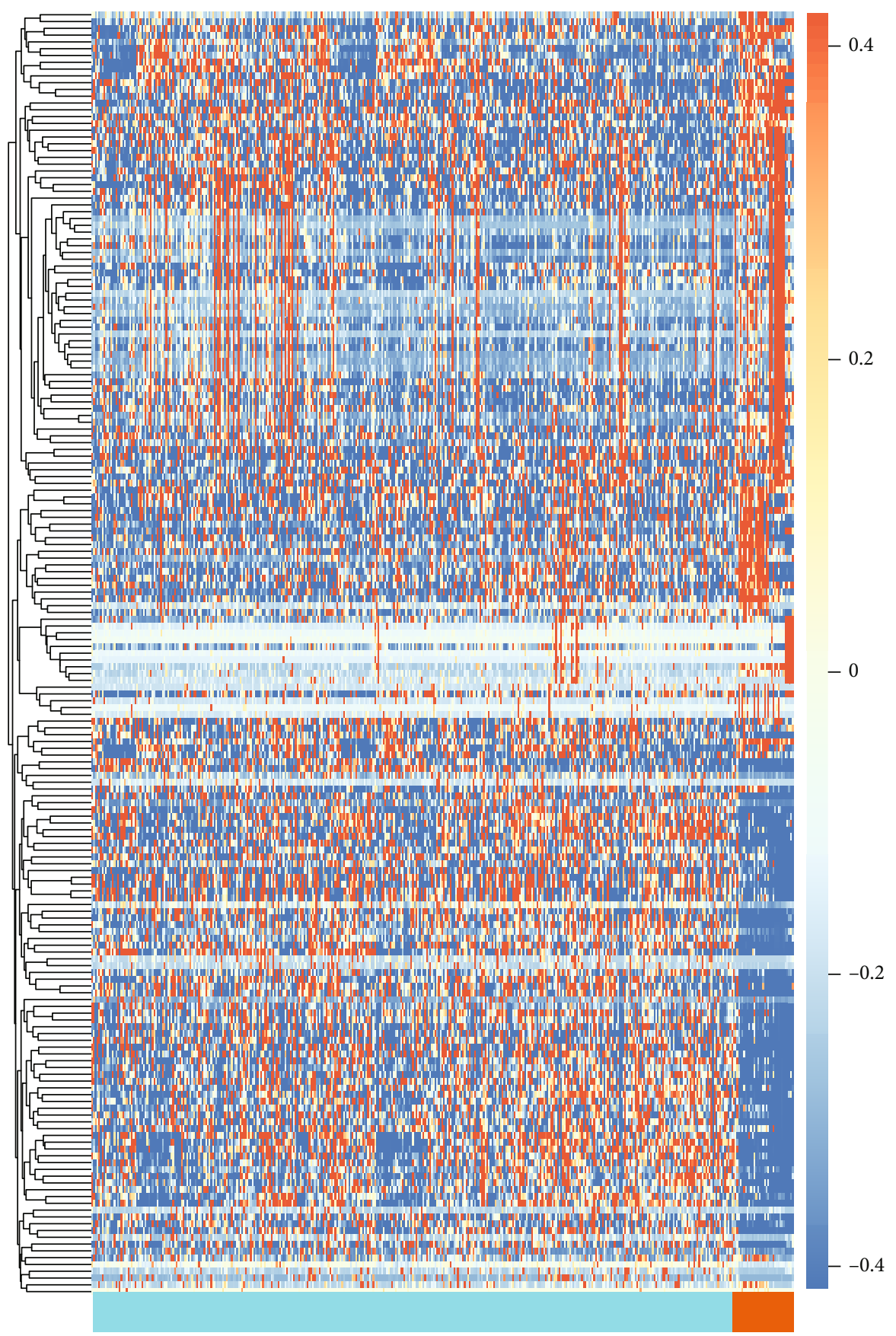

(a)

Figure 1: Continued. 


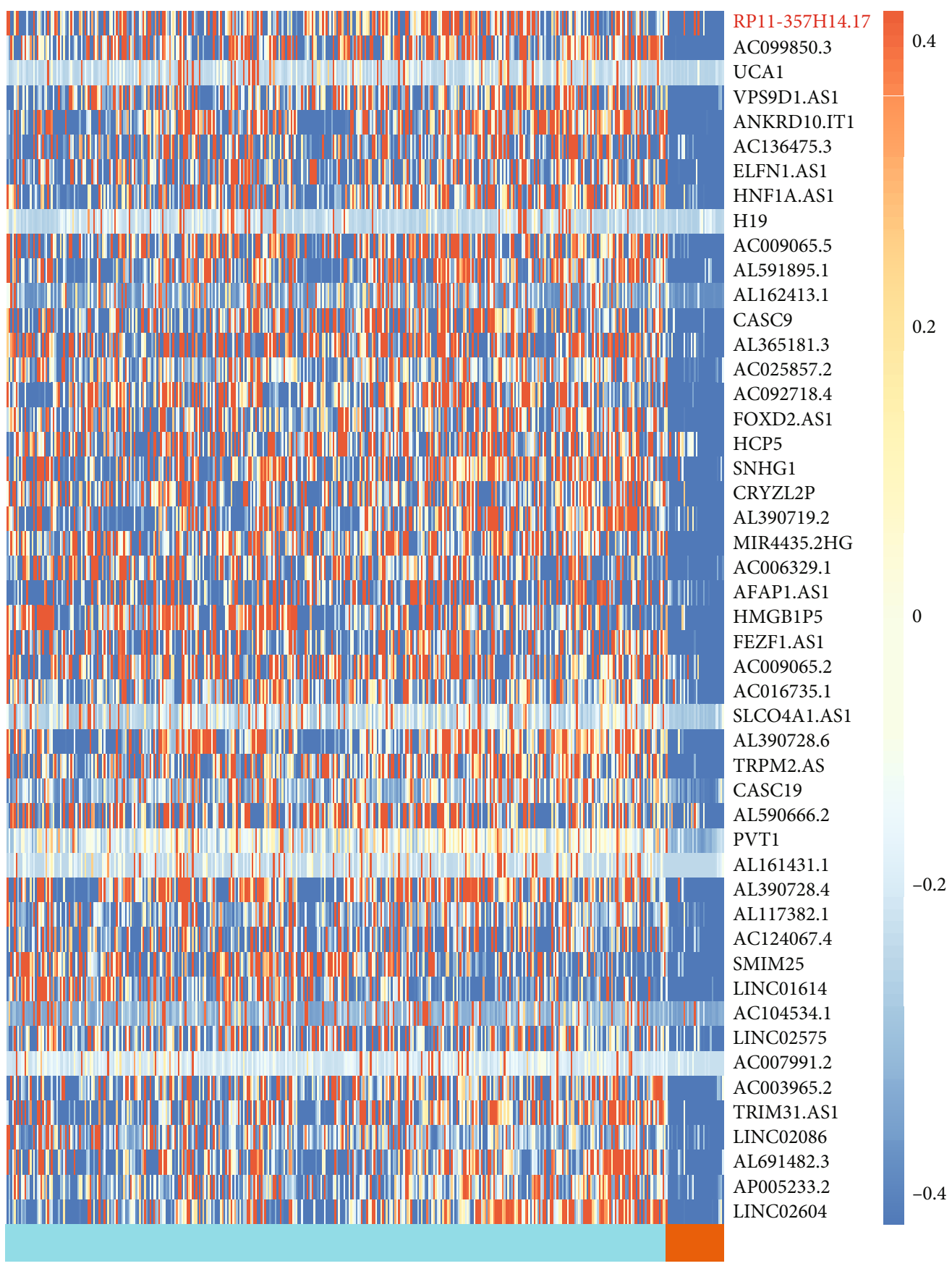

(b)

FIgure 1: Continued. 


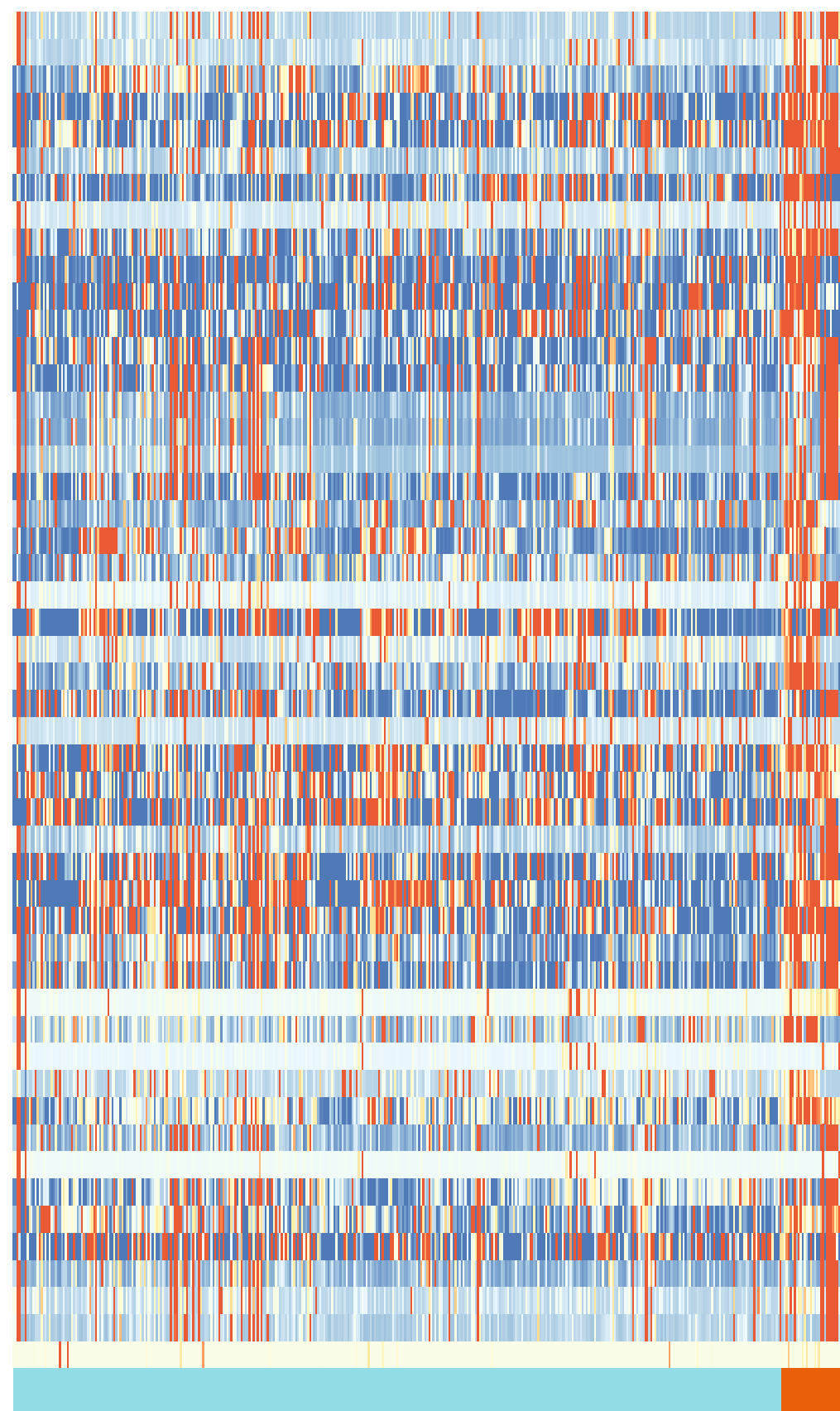

Normal

Cancer

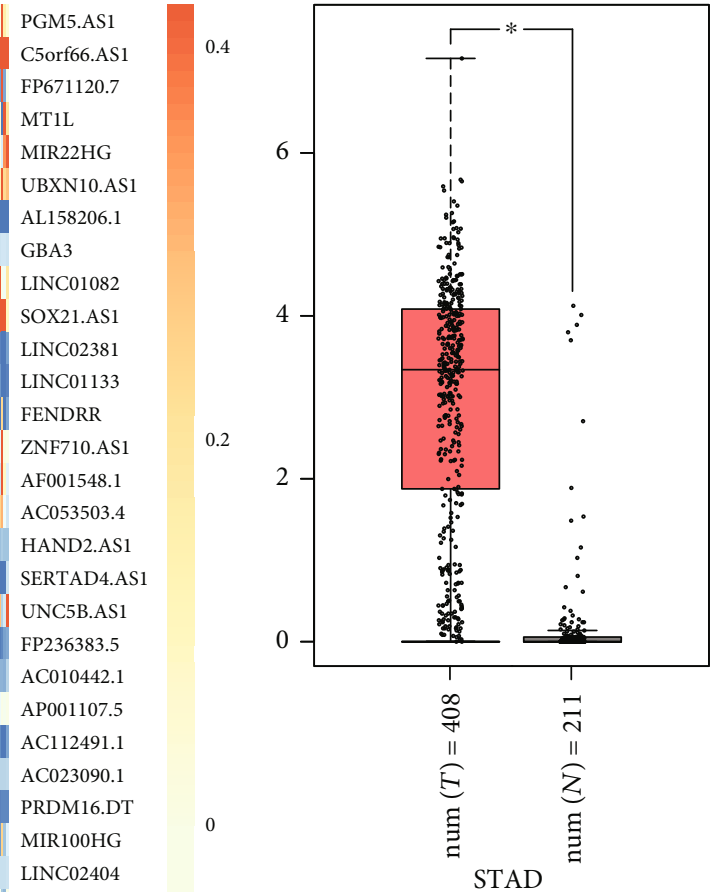

HEIH

TP53TG1

AC243919.1 NALT1 AC105942.1 AL391121.1 TRIM52.AS1 AC008808.2 ZNF667.AS1 AC103563.7 AC104699.1 CYP4F29P LINC00261 RAB11B.AS1 MBNL1.AS1 HCG22 AC011472.1 GGTAIP AC015922.2 CARMN PART1 AC036108.3 PNLIPRP2 $-0.4$ (c)

Figure 1: Continued. (d) 


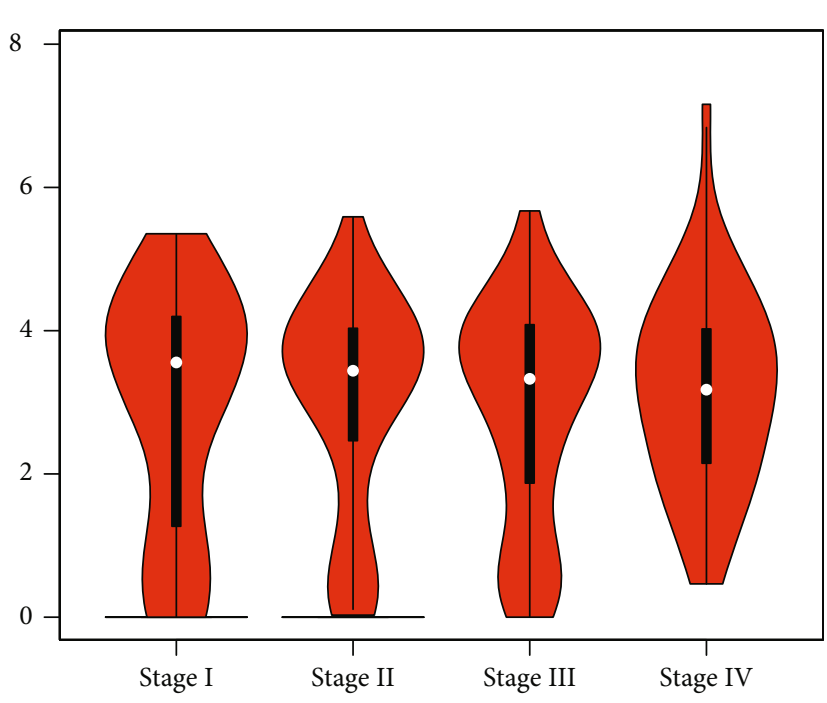

$F$ value $=0.385$

$\operatorname{Pr}(>F)=0.764$

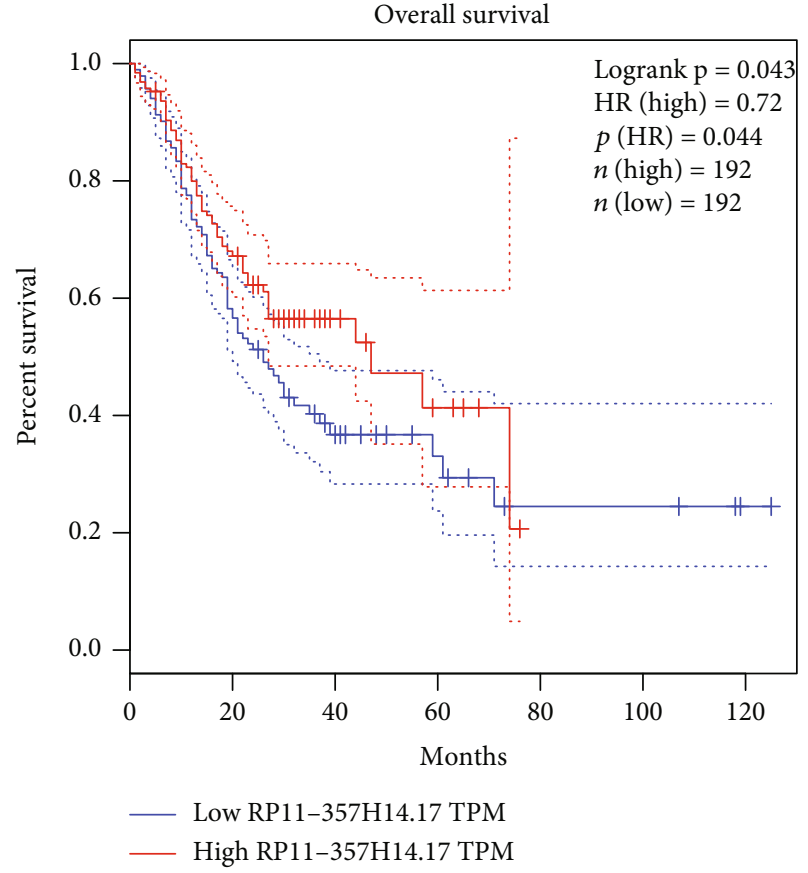

(f)

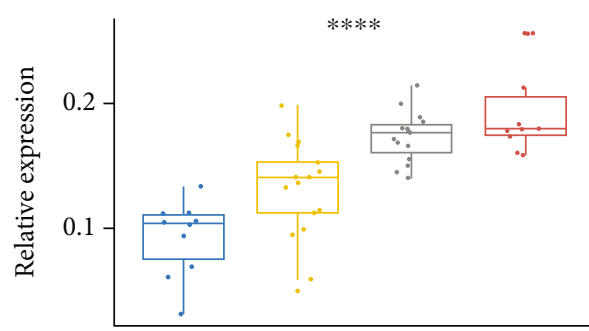

Stage
田 I
亩 III
畾 II
审 IV

(g)

Figure 1: Continued. 


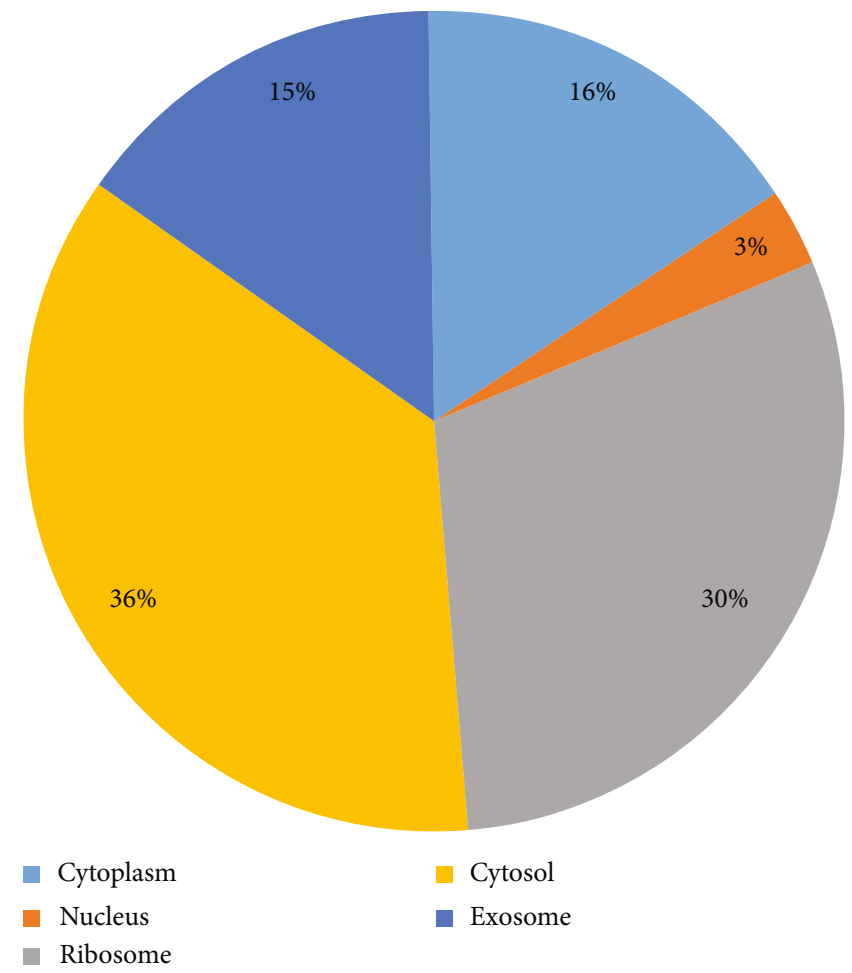

(h)

FIGURE 1: RP11-357H14.17 is overexpressed in human stomach cancer. (a) Heatmap of the all significantly differently expression lncRNAs $(n=3790$, cancer vs. normal, adj.p < 0.05). (b, c) Heatmaps of TOP 50 up and down-regulated significantly differently expressed lncRNAs; RP11-357H14.17 was highlighted with red font in (b). For $(\mathrm{a}-\mathrm{c})$, rows denote lncRNAs, and columns represent patients. Blue to red indicates a trend from low to high expression. Boxplot of comparison between cancer and normal control tissues (d) and cancer tissues with different TMN stages (e) in RP11-357H14.17 expression; the data is provided by TCGA-STAD. (f) Comparison of overall survival between RP11-357H14.17 high expression and low expression group; the data is provided by TCGA-STAD. (g) Boxplot of comparison between cancer and normal control tissues (left) and cancer tissues with different TMN stages for 52 paired human GC tissues (right). ${ }^{* * * *} p<0.0001$, for the left panel, unpaired $t$ test, and for the right panel, one-way ANOVA. (h) Prediction of subcellular location of RP11357H14.17 provided by online tool lncLocator.

the Polycomb Group protein (PcG) complex to induce histone trimethylation and regulate $\mathrm{mRNA}$ expression of related genes at the transcriptional level [13]. At the same time, lncRNAs directly bind to promoters and regulate gene expression [14]. In cytoplasm, IncRNA also binds directly to mRNA, affecting its stability and expression at the transcriptional level [3]. Meanwhile, cytoplasmic lncRNAs can regulate gene expression in a posttranscriptional level. As previously mentioned, lncRNAs, together with miRNAs, act as sponges and induce a "ceRNA" to regulate gene expression [3]. On the other hand, lncRNA also affects the stability of proteins and inhibits their expression at the posttranscriptional level [13]. In the present study, by analyzing the TCGA-STAD data, we found that RP11-357H14.17 is the most up-regulated within human gastric cancer. Also, through an online predicting tool, lncLocator, we found that RP11-357H14.17 was located in cytosol and ribosome, which implied that most likely RP11-357H14.17 can be affected by sponges miRNA to regulated certain gene. So far, only two researches implied the role of RP11-357H14.17 in human cancers $[15,16]$. One of the researches is concerning its role in diffuse-type gastric carcinoma (DGC). Overexpression of
RP11-357H14.17 was spotted in human DGC; its expression were associated with increased tumor size, deeper depth of invasion, lymphatic metastasis, and advanced pathological stage [15]; this results was similar to our finding.

By using ssGSEA on c6 gene sets, we found that RP11$357 \mathrm{H} 14.17$ was associated with enhanced ATF2 signaling. Activated transcription factor 2 (ATF2) is a member of the activated protein 1 (AP-1) transcription factor family that contains DNA-binding proteins in basic Leucine Zipper (bZIP) $[17,18]$. The N-terminal zinc finger region and the deactivation region activate the transcriptional activity of ATF2, while the C-terminal tissue has homologous dimerization and heterodimerization [19]. Its heterodimer partners are JUN, FOS, CREB, and MAF, leading to the formation of the AP-1 transcription factor [17]. ATF2 can also act as an epigenetic regulator, acting as a histone acetyltransferase (HAT), specifically acetylating histones $\mathrm{H} 2 \mathrm{~B}$ and $\mathrm{H} 4$, and triggering its own DNA binding effect [17]. The transcriptional functions of ATF2 show a wide range of target genes, which can be grouped into the following categories including cell cycle, immune and inflammatory responses, AP-1 binding partner, and apoptosis [19]. Our results also indicated 


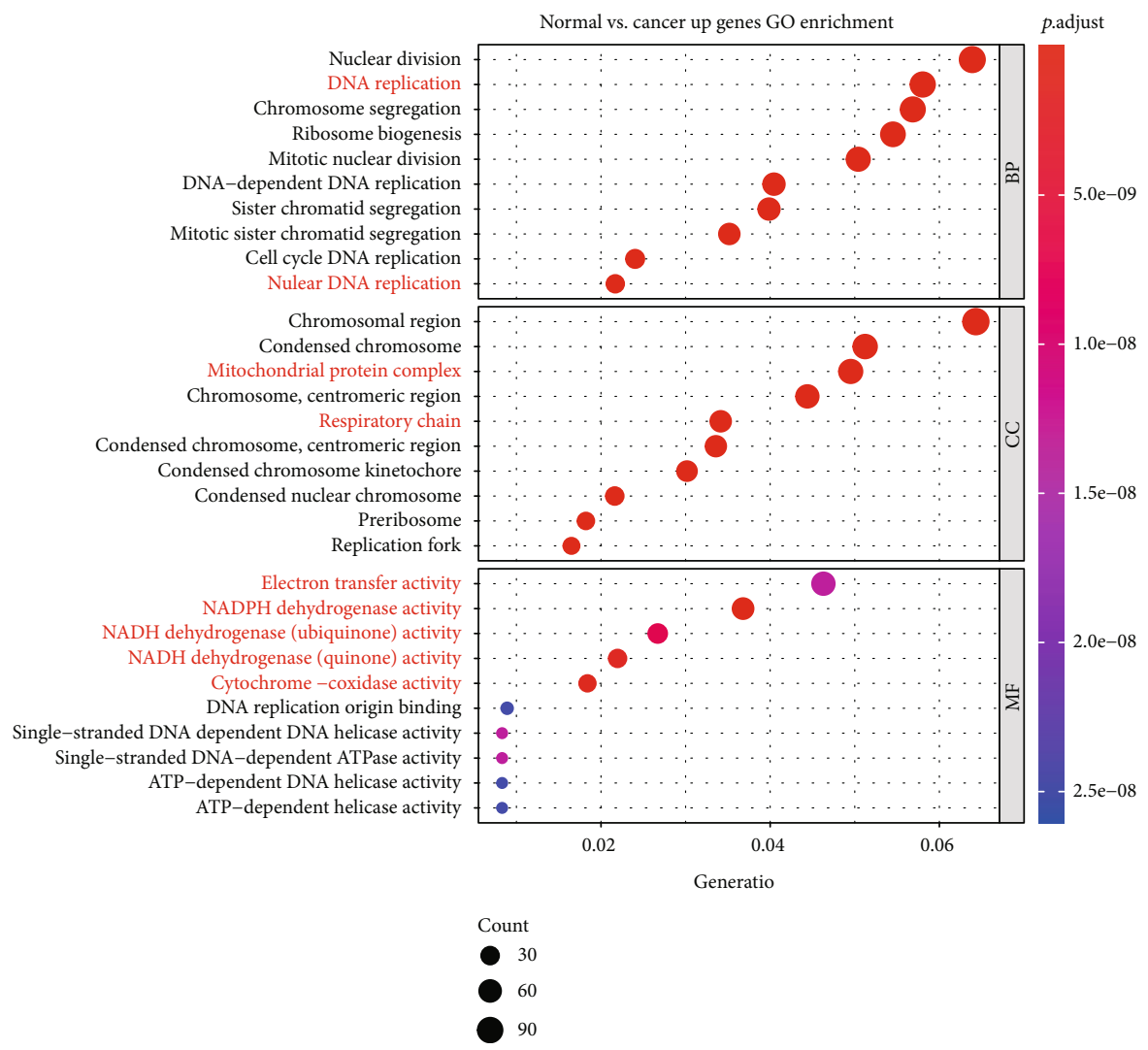

(a)

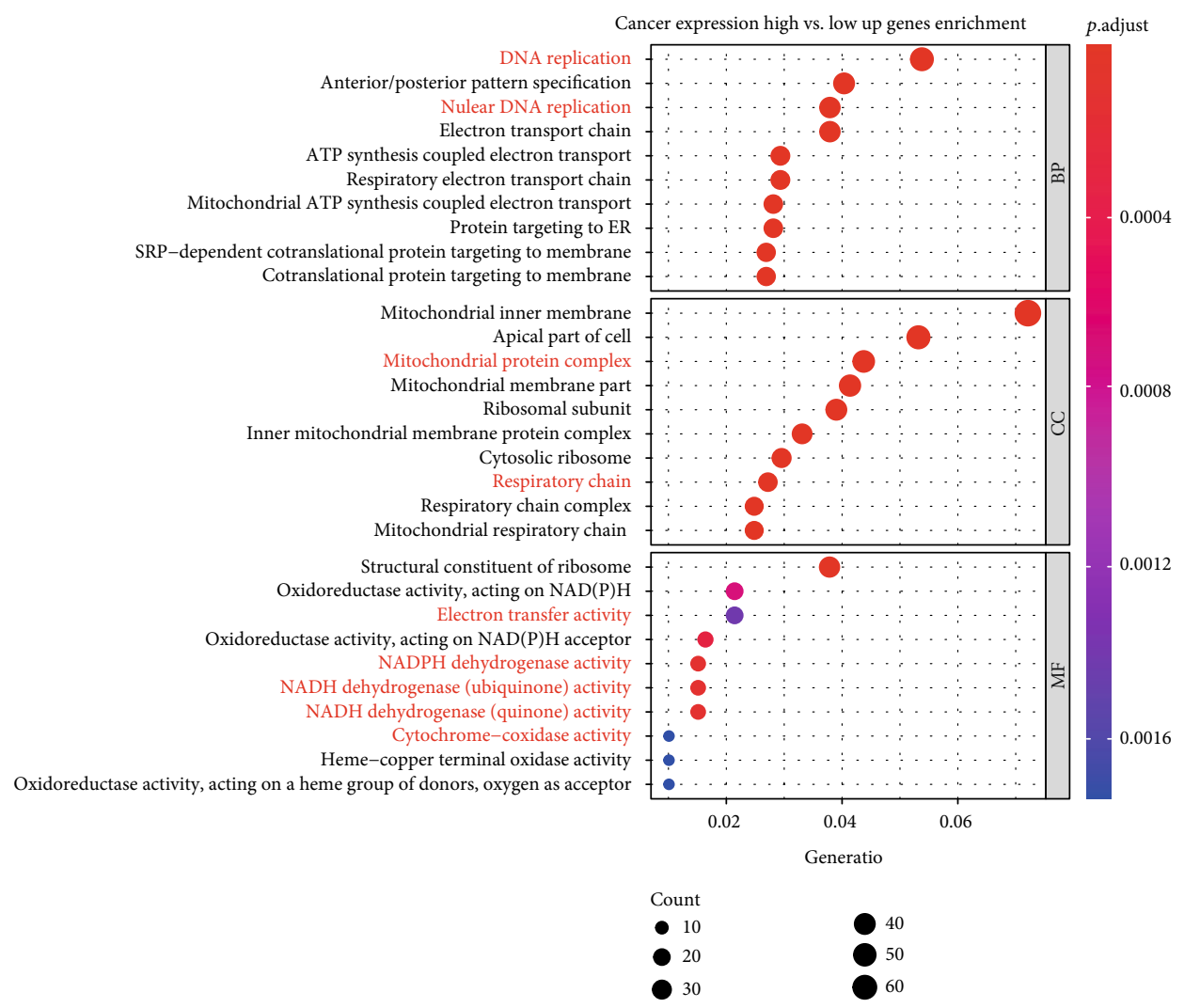

(b)

Figure 2: Continued. 


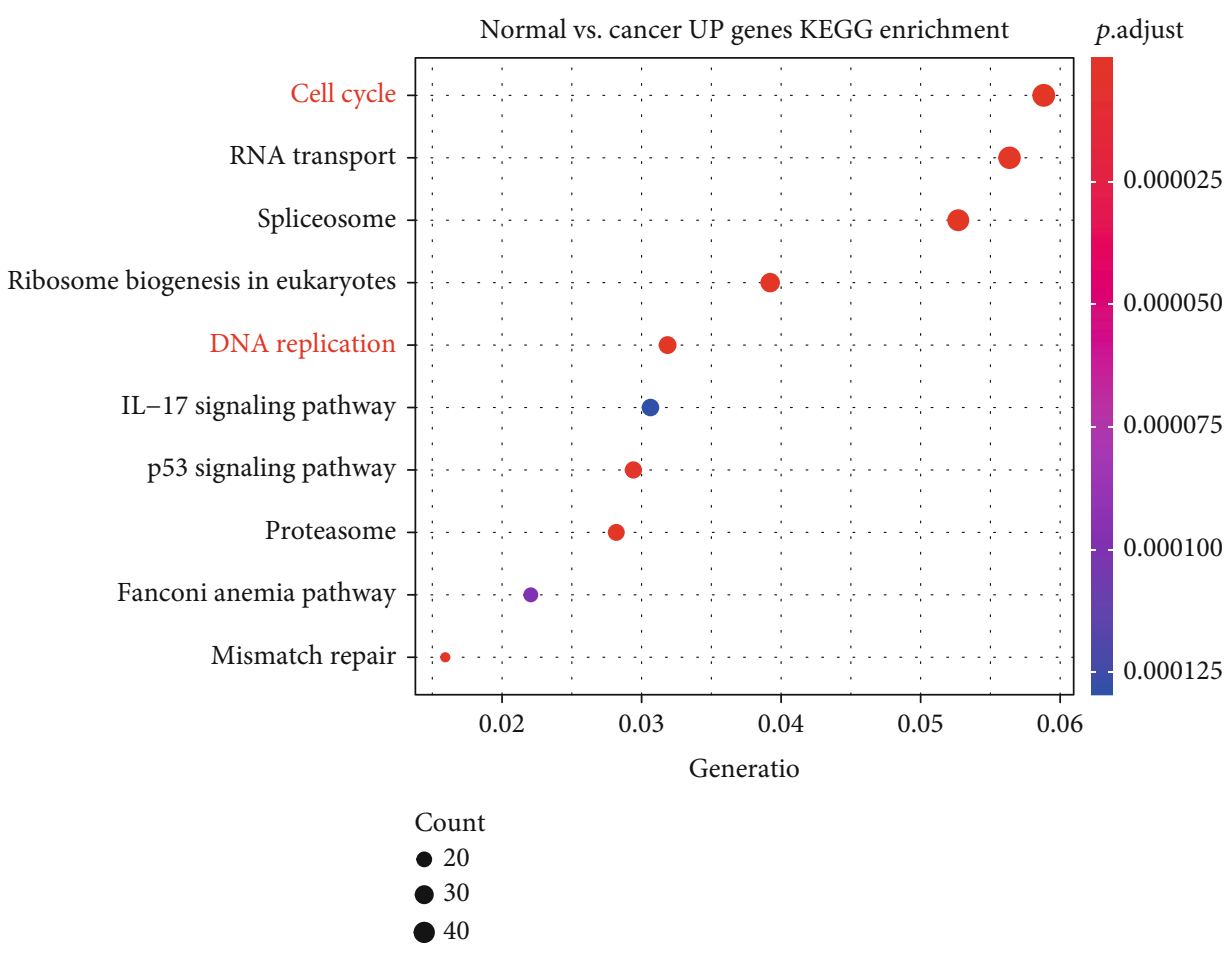

(c)

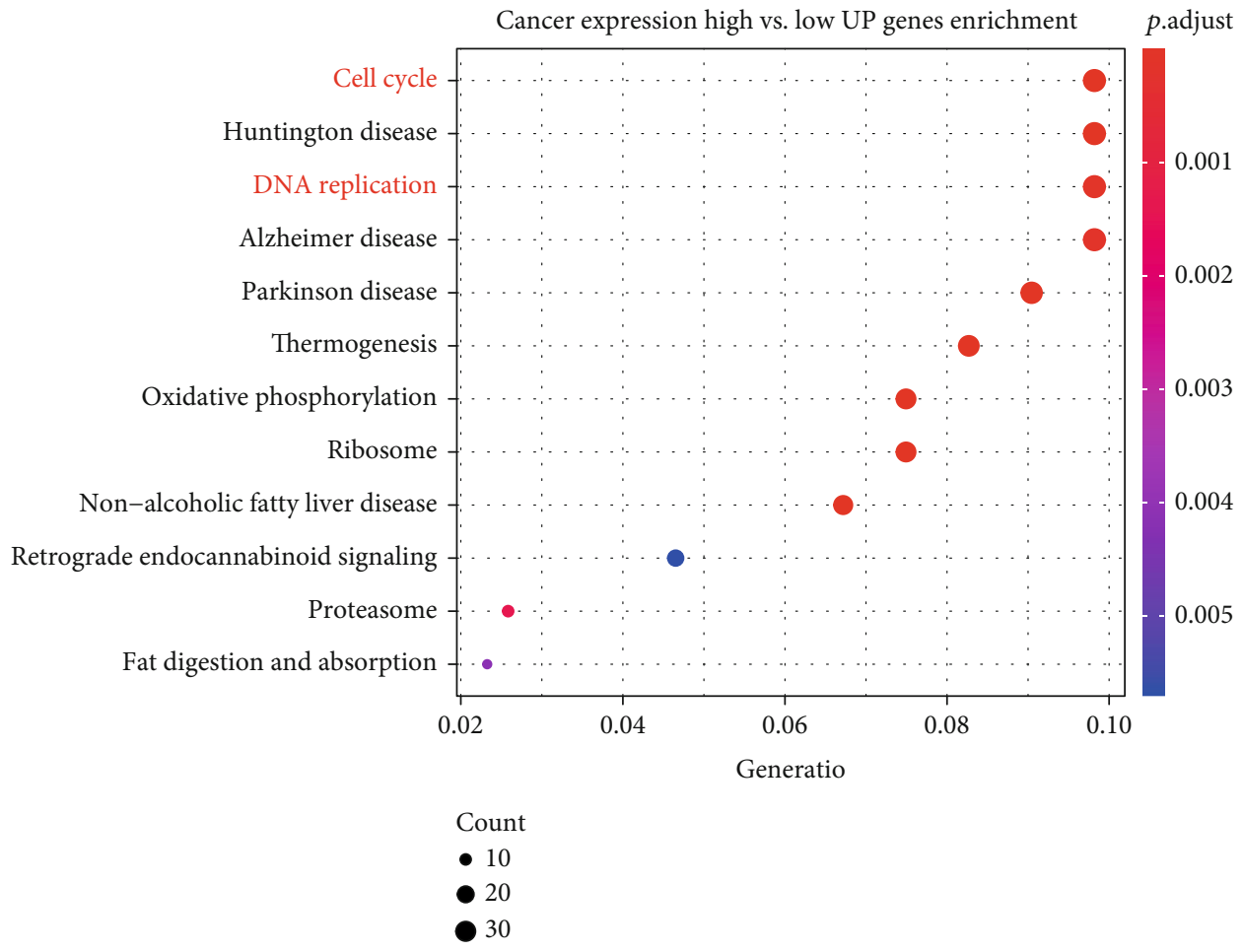

(d)

FIgURE 2: Continued. 


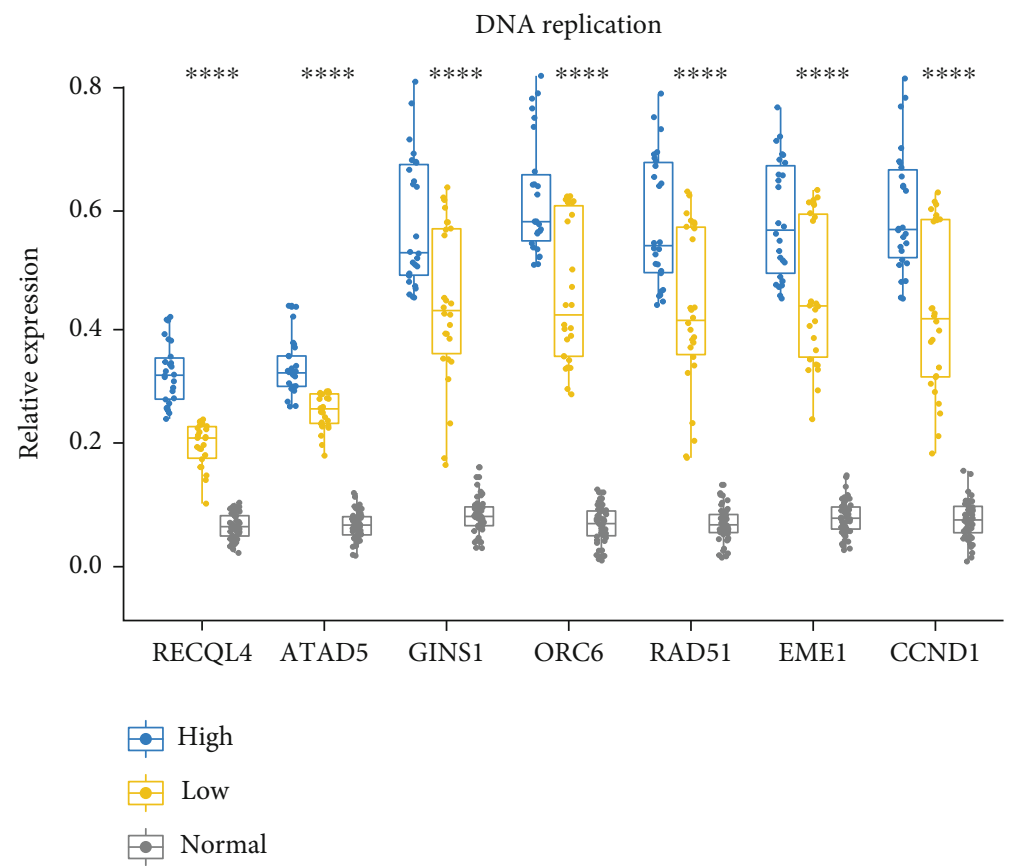

(e)

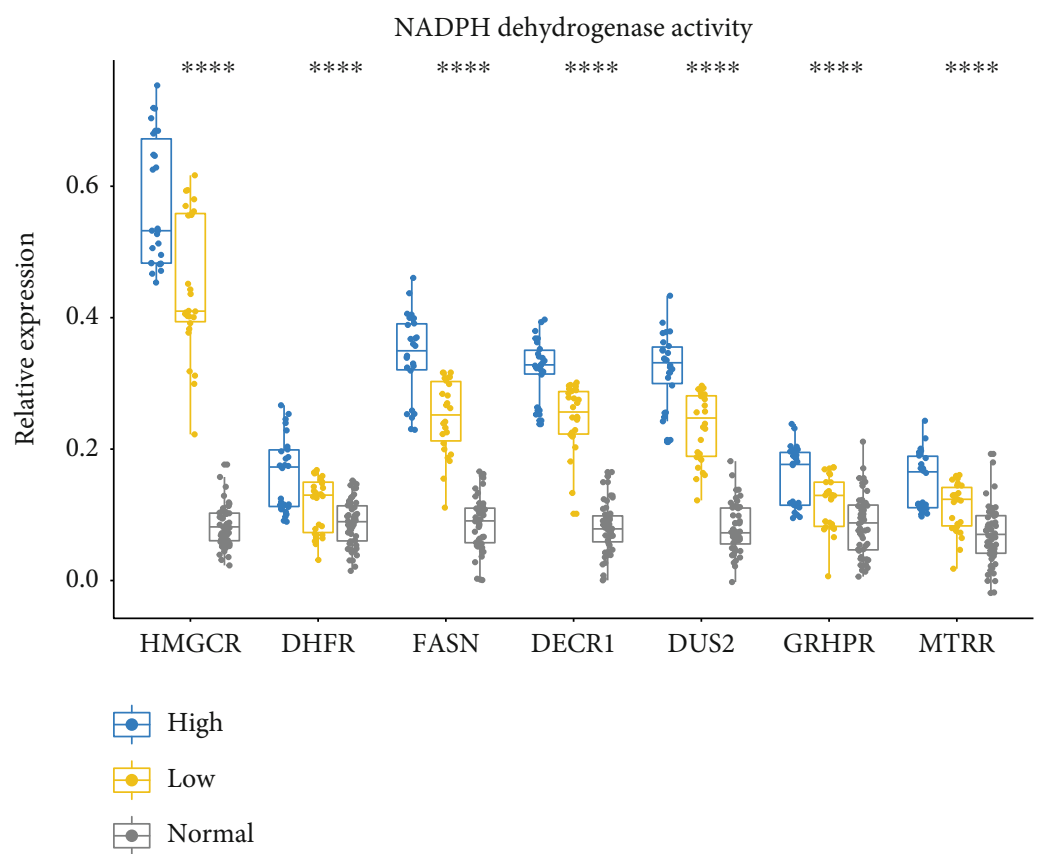

(f)

FIGURE 2: Increased expression of RP11-357H14.17 is associated with increased DNA replication and metabolism in human gastric cancer. GO enrichment was performed using significantly up-regulated genes compared between cancer and normal controls (a) and RP11357H14.17 high and low expression sample sets (b). KEGG enrichment was performed using significantly up-regulated genes compared between cancer and normal controls (c) and RP11-357H14.17 high and low expression samples from TCGA-STAD (d). Representative genes expression stands for "DNA replication" (e) and "NADPH dehydrogenase activity" (f). GO terms was determined by real-time PCR. ${ }^{* * * *} p<0.0001$, one-way ANOVA.

that ATF downstream genes including KRT8, PARD6B, THOC6, and WT1 were well correlated to RP11-357H14.17 expression. The detailed mechanism is worthy to be further explored in the future. Besides the regulation by ATF2 on the genes indicated above, a previous study revealed that anisomycin, a potent activator of ATF2, and JNK, induces expression of FoxP3 in both normal and malignant mammary epithelial cells [20]. This result might also implied that 


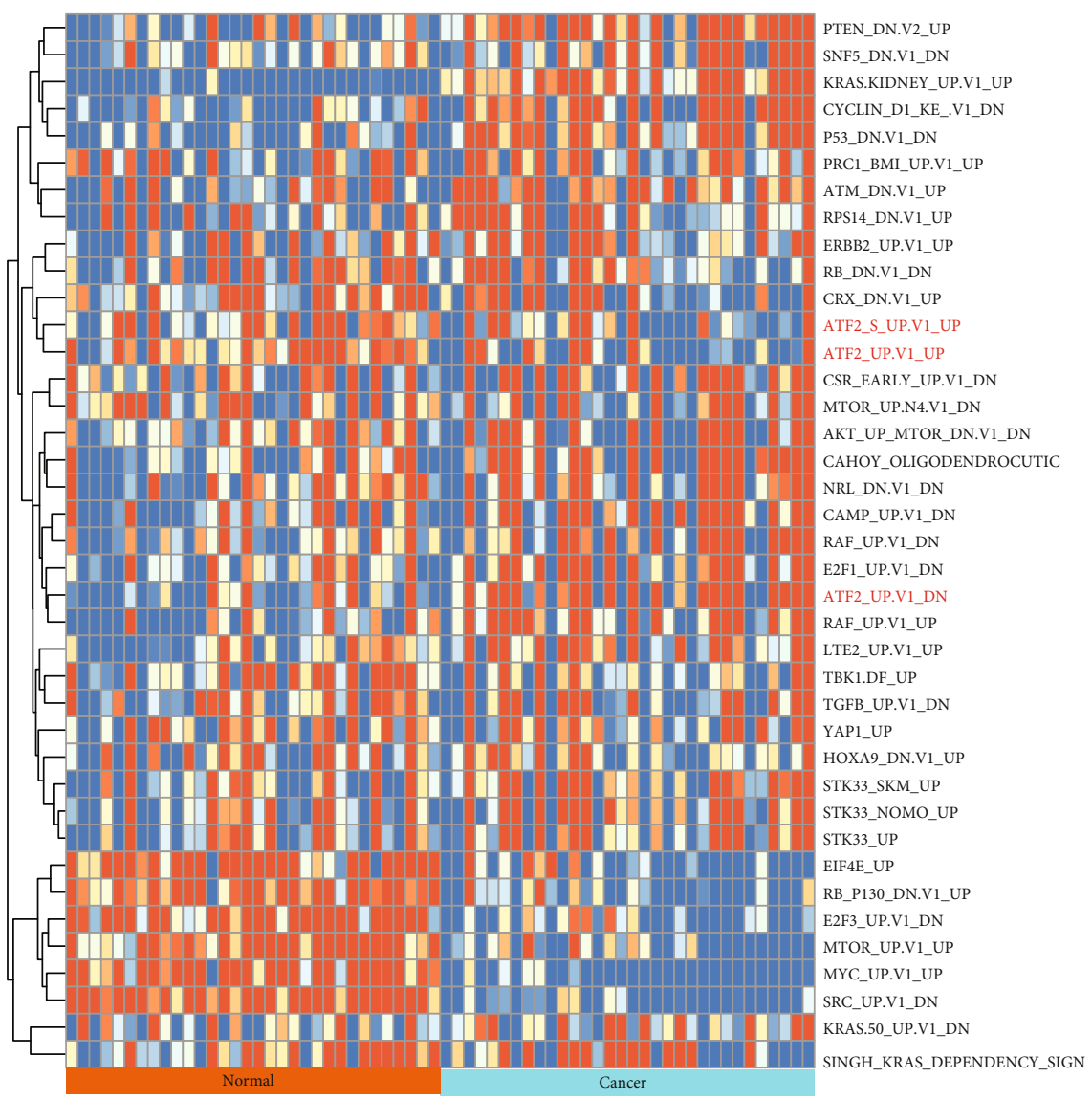

(a)

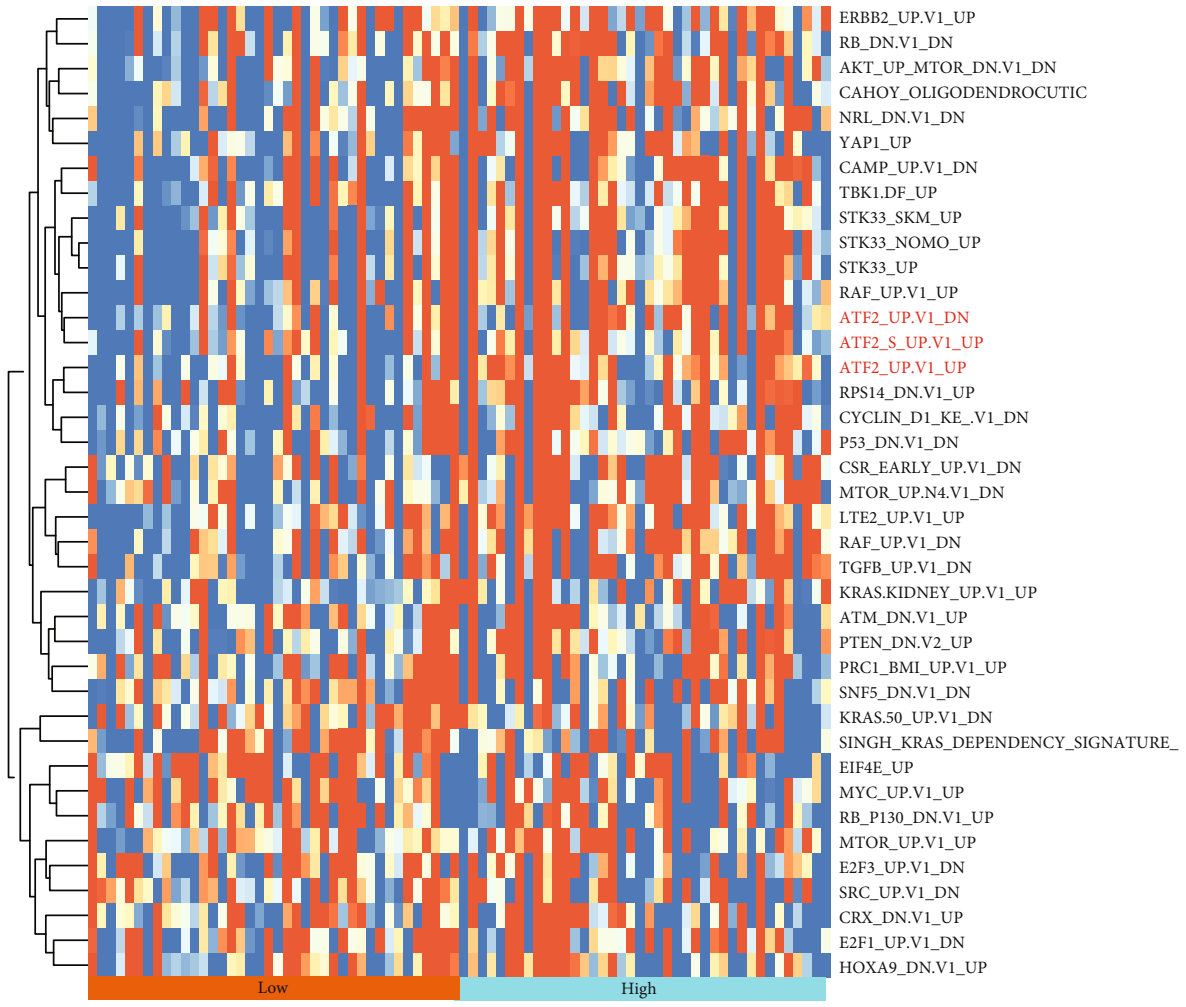

(b)

FIgure 3: Continued. 


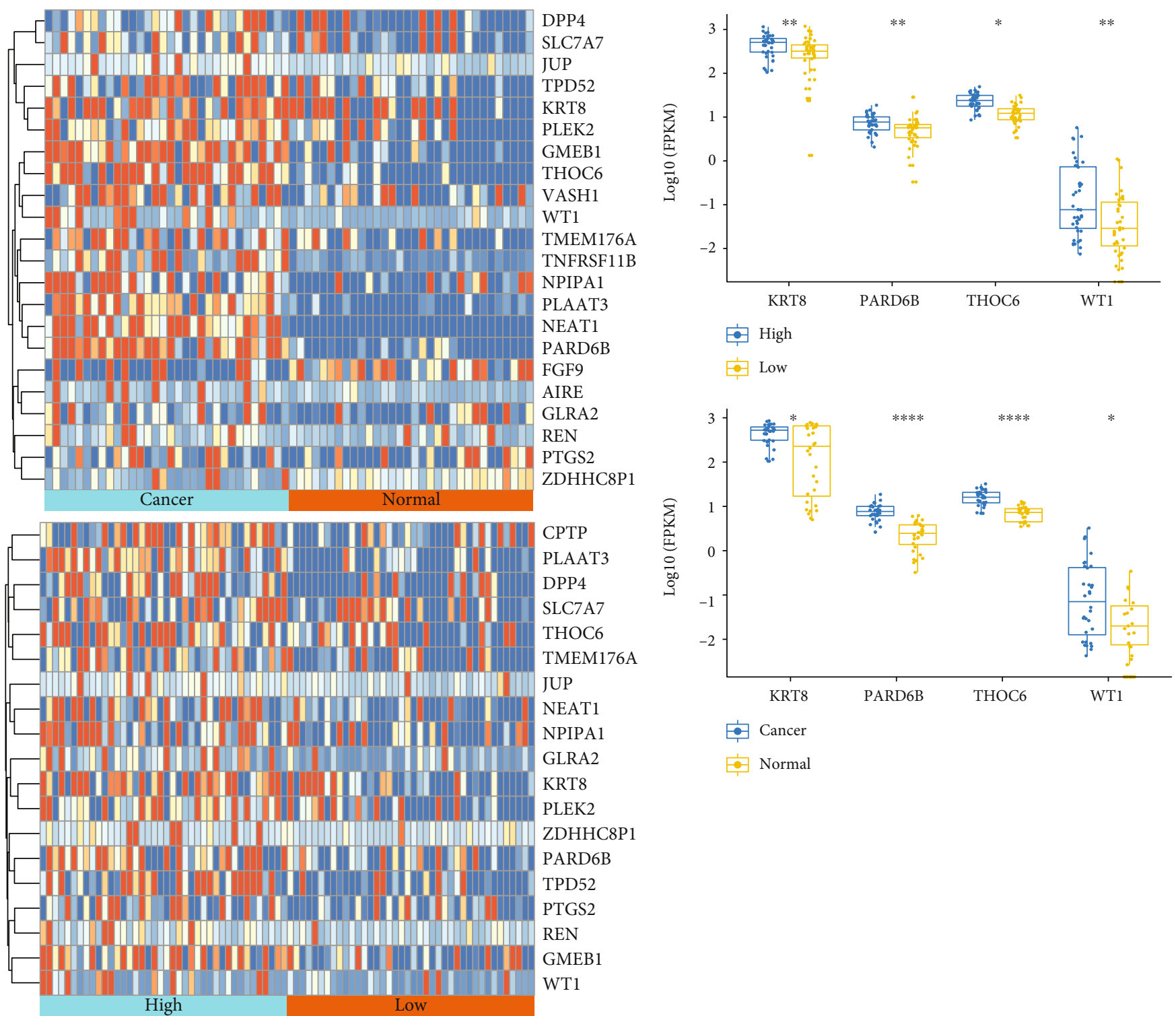

(c)

(d)

Figure 3: Continued. 


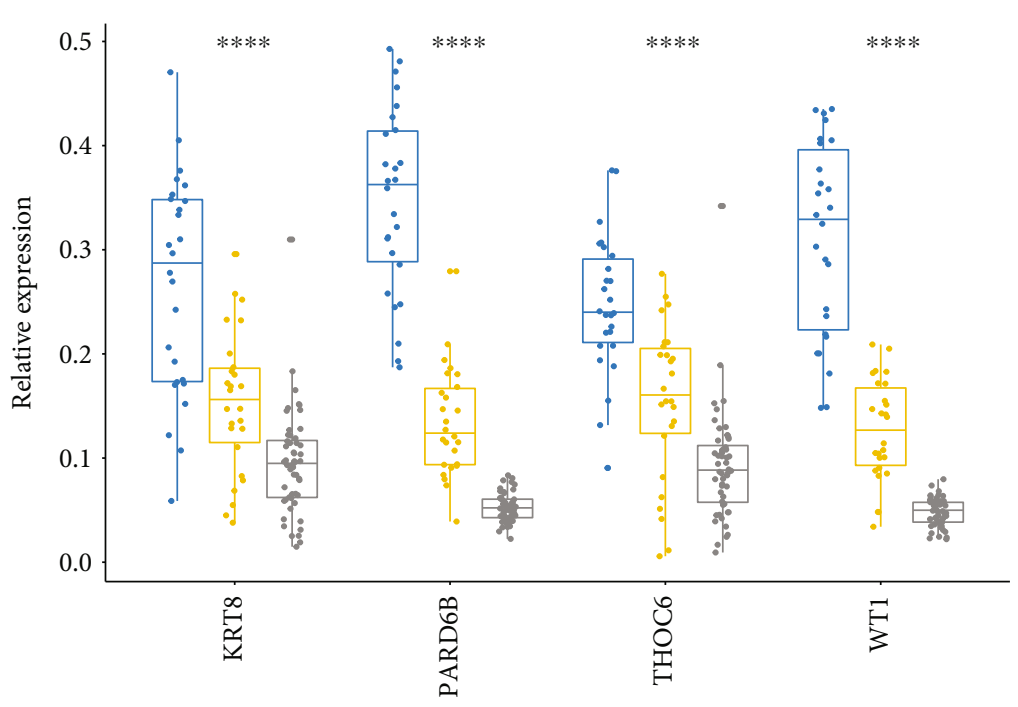

$$
\begin{aligned}
& \text { High } \\
& \text { Low }
\end{aligned}
$$

(e)
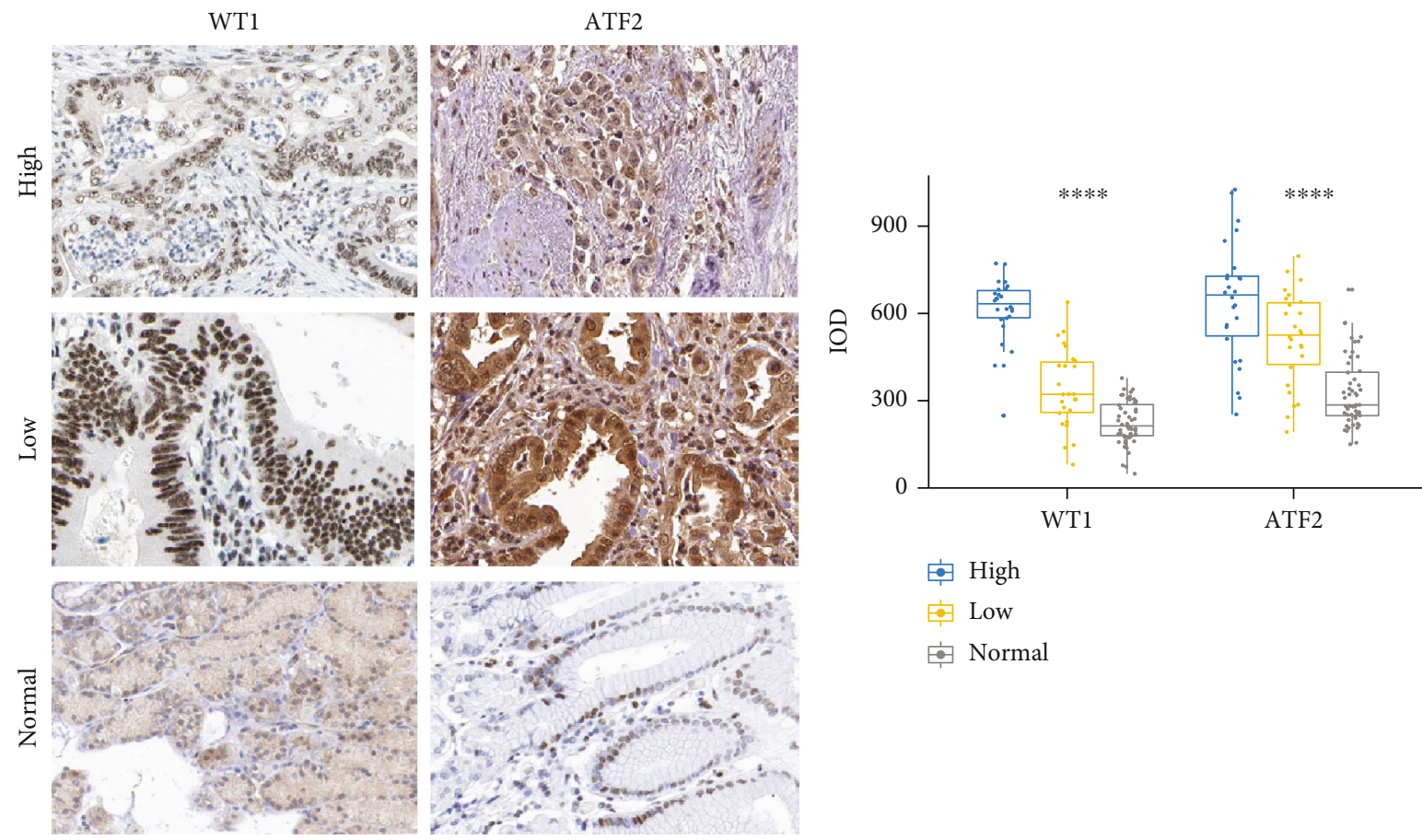

$$
\begin{aligned}
& \text { High } \\
& \text { Low } \\
& \text { Normal }
\end{aligned}
$$

(f)

FIGURE 3: RP11-357H14.17 promotes the malignancy by promoting ATF2 signaling in human gastric cancer. Heatmap of all significantly different gene sets of c6 ssGSEA analysis for comparison between cancer and normal control samples (a) and RP11-357H14.17 high and low expression samples (b) from TCGA-STAD. ATF2-related gene sets were highlighted with red font (c). Heatmap of up-regulated genes in "ATF2_up.v1_DN" gene set derived from comparison between cancer and normal control samples and RP11-357H14.17 high and low expression samples from TCGA-STAD. The representative genes stand for “ATF2_up.v1_DN" gene set were highlighted in bold. (d) Representative gene expression stands for "ATF2_up.v1_DN" gene set was compared between cancer and normal control samples, RP11$357 \mathrm{H} 14.17$ high and low expression samples from TCGA-STAD, ${ }^{*} p<0.05,{ }^{* *} p<0.01$, and ${ }^{* * * *} p<0.0001$, by unpaired $t$ test, and 52 paired GC tissues categorized as RP11-357H14.17 high, low expression, and normal control, ${ }^{* * * *} p<0.0001$, one-way ANOVA (e). (f) Representative IHC staining figures for ATF2 and WT1 protein and comparison between 52 paired GC tissues categorized as RP11$357 \mathrm{H} 14.17$ high, low expression, and normal control, ${ }^{* * * *} p<0.0001$, one-way ANOVA. 


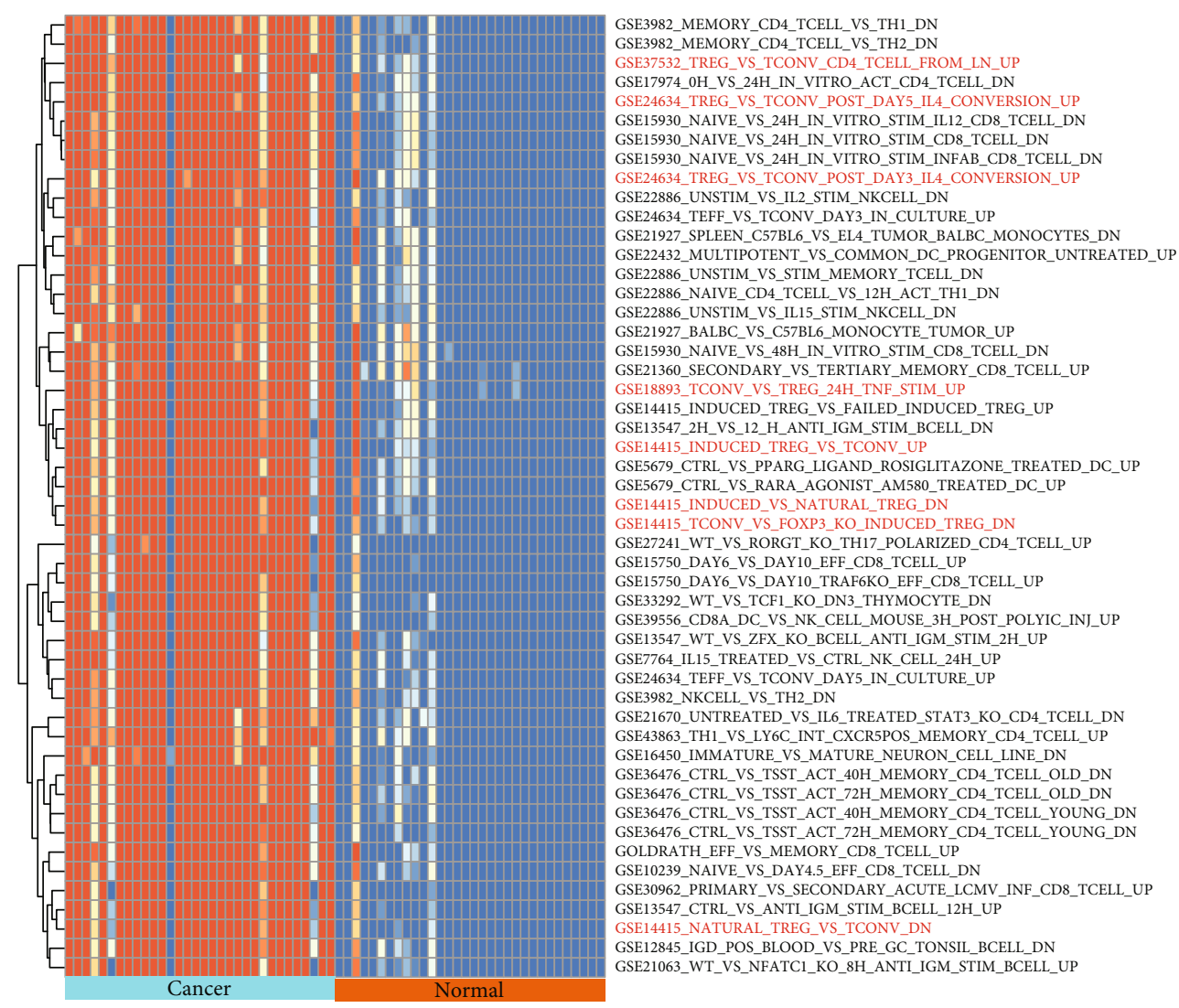

(a)

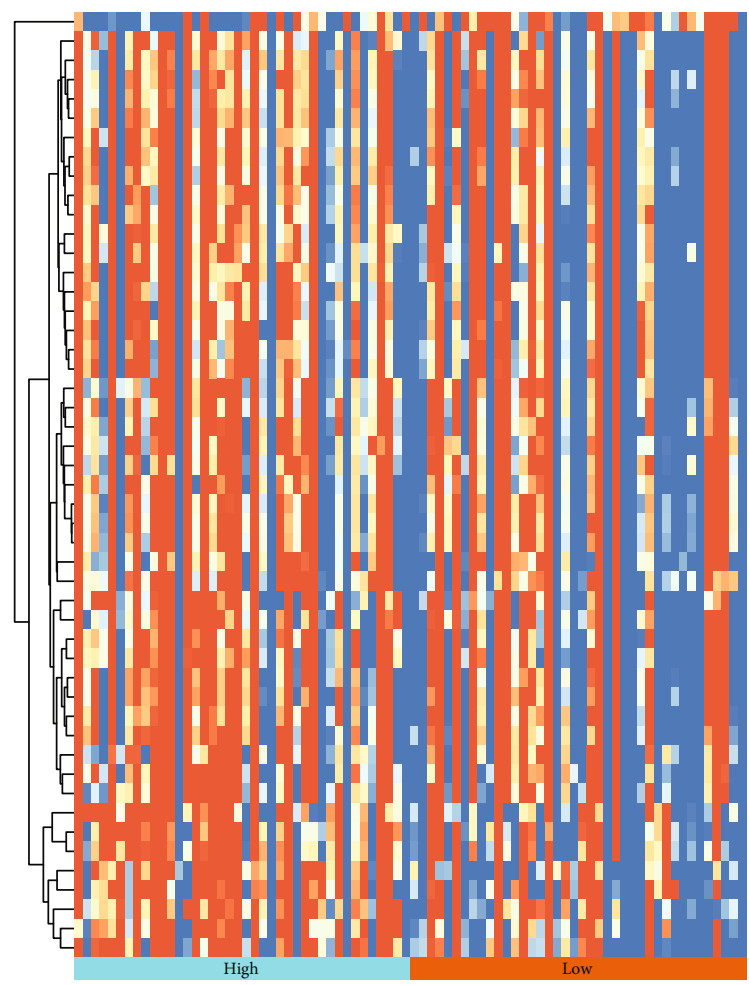

GSE35825_UNTREATED_VS_IFNG_STIM_MACROPHAGE_DN GOLDRATH_EFF_VS_MEMORY_CD8_TCELL_UP

(CSE28726_NAIVE_VS_ACTVATED_CD4_TCELL_DN

GSE36476_CTRL_VS_TSST_ACT_40H_MEMORY_CD4_TCELL_OLD_DN GSE36476_CrRL_VS_TST_AC_40H_MEMORY_CD4_TCELL_YOUNG_DN GSE7568_LA VS IL_ AND_DEXAMETHASONE TREATED MACROPHANE GSE5679 CTRL_VS PPARG LIGAND ROSIGLITAZONE TREATED DC UP GSE5679_CTRL_VS_RARA_AGONIST_AM580_TREATED_DC_UP GSE14415_INDUCED_VS_NATURAL_TREG_DN

GSE14415_TCONV_VS_FOXP3_KO_INDUCED_TREG_DN

GSE13547_2H_VS_12_H_ANTI_IGM_STIM_ZFX_KO_BCELL_DN

GSE13547_WT_VS_ZF_KO_BCELL_ANT__GM_STIM_2H_UP

_CD 4 TCELL DN GSE33292 WT VS TCF1 KO DN3 THYMOCYTE_DN GSE39110 DAY3 VS DAY6 POST IMMUNIZATION CD8 TCELL_DN GSE15750_DAY6_VS_DAY10_EFF_CD8_TCELL_UP

GSE15750_DAY6 VSDAY10 TRAFKO EFE_CD8_TCELL UP GSE22886_NAIVE_CD4_TCELL_VS_48H_ACT_TH1_DN

GSE14415_INDUCED_TREG_VS_FAILED_INDUCED_TREG_UP

GSE21360_SECONDARY_VS_TERTIARY_MEMORY_CD8_TCELL_UP GSE22886_UNSTIM VS _L15_STIM_NCEDLDNO

GSE22886_UNSTIM_VS_IL_15_STIM_NKCELL_DN GSE15930 NAIVE VS 24H IN VITRO STIM_L12 CD8 TCELL DN

GSE15930_NAIVE_VS_24H_IN_VITRO_STIM_CD8 TCELL_DN

GSE15930_NAIVE_VS_24H_IN_VITRO_STIM_INFAB_CD8_TCELL_DN GSE16450_IMMATURE_VS_MATURE_NEURON_CELL_LINE_DN GSE37532_TREG_VS_TCONV_CD4_TCELL_FROM_LN_UP GSE14699_DELETIONAL_TOLERANCE_VS_ACTIVATED_CD8_TCELL_DN GSE23568_CTRL_VS_ID3_TRANSDUCED_CD8_TCELL_DN GSE13547_2H_VS_12_H_ANTI_IGM_STIM_BCELL_DN GSE14415_INDUCED_TREG_VS_TCONV_UP GSE1983_CCON__VS_TREG_24H_TNF_STAM_UP MAST_CEL_UP GSE24210_CTRL_VS_A3R_INHIBITOR_TREATED_MAST_CELL_UP GSE14415_ACT_VS_CTRL_NATURAL_TREG_UP F_CD8_TCELL_UP

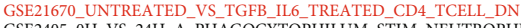
GSE2405 $0 H_{-V S}$ - $9 H$ _ A PHAGOCYTOPHIUM_STIM_NEUTROPHIL_UP GSE14000_TRANSLATED_RNA_VS_MRNA_4H_LPS_DC_DN GSE14000_TRANSLATED_RNA_VS_MRNA_DC_DN GSE17580_UNINFECTED_VS_S_MANSONI_INF_TREG_DN GSE26030_TH1_VS_TH17_RESTIMULATED_DAY5_POST_POLARIZATION_UP GSE39820_TGFBETA1_IL6_VS_TGFBETA1_IL6_IL23A_TREATED_CD4_TCELL_DN

(b)

Figure 4: Continued. 


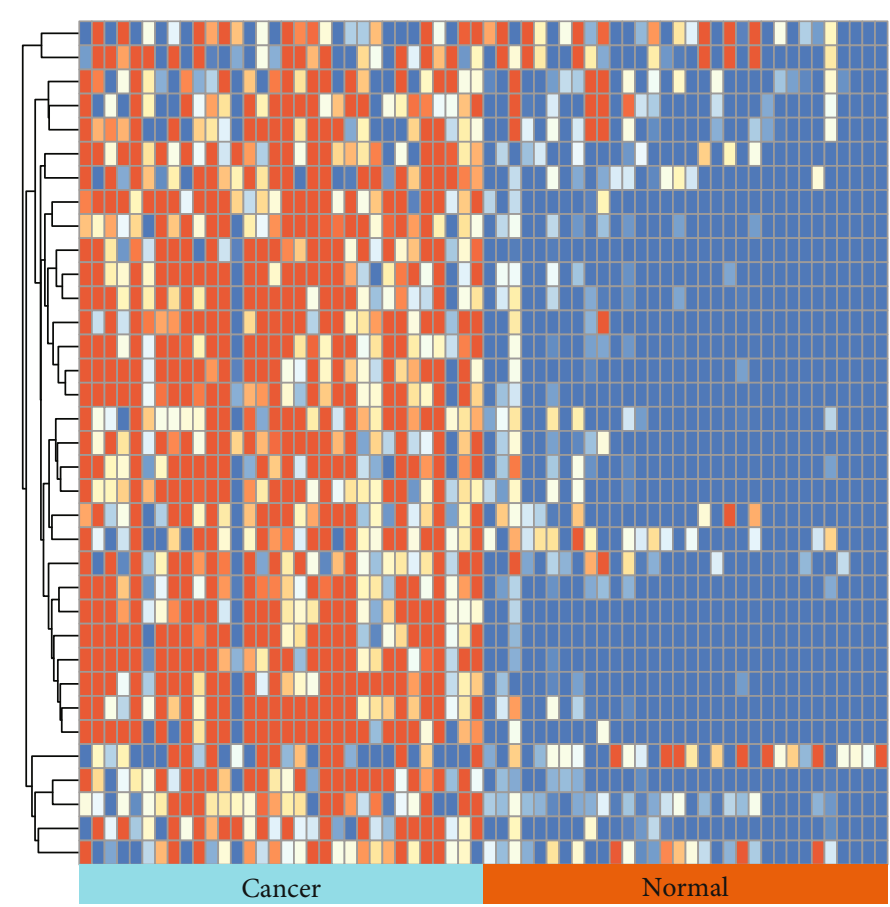

PYCARD DUT

DCK

HAUS1

FOXP3

NME1

PBK

TRIP13

CDC25C

IKZF2

CKS1B

CDK1

NUF2

SGO1

PLK1

CDKN3

AURKB

CDC20

CDCA3

SHMT2

HAT1

RAD51AP1

RFC3

DSCC1

TROAP

KIF18B

CCNB2

MAD2L1

PHGDH

HIST1H2B

HIST

LY6E

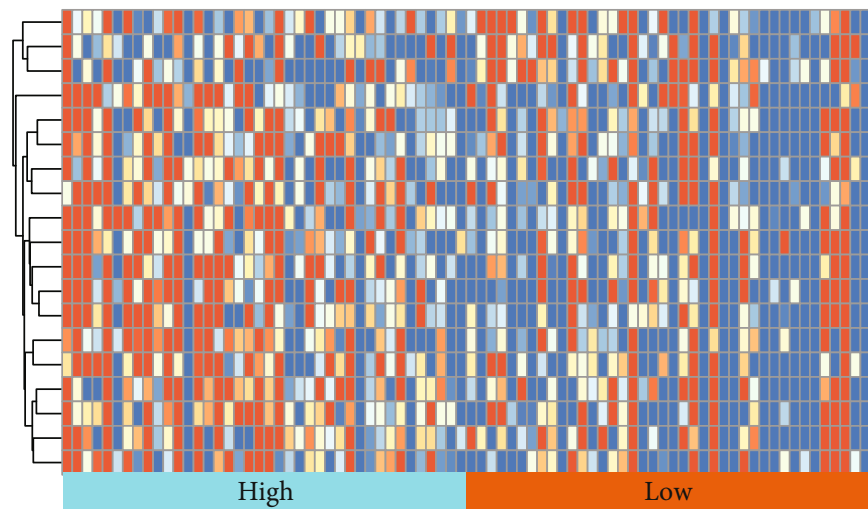

(c)
HEATR3

DUT

HATI

RAD51AP

RFC3

CDKN3

POLE2

SPDL1

SGO1

CDK1

FAM72A

NUF2

FOXP3

DSCC1

CCNB2

IKZF2

KIF18B

PLK1
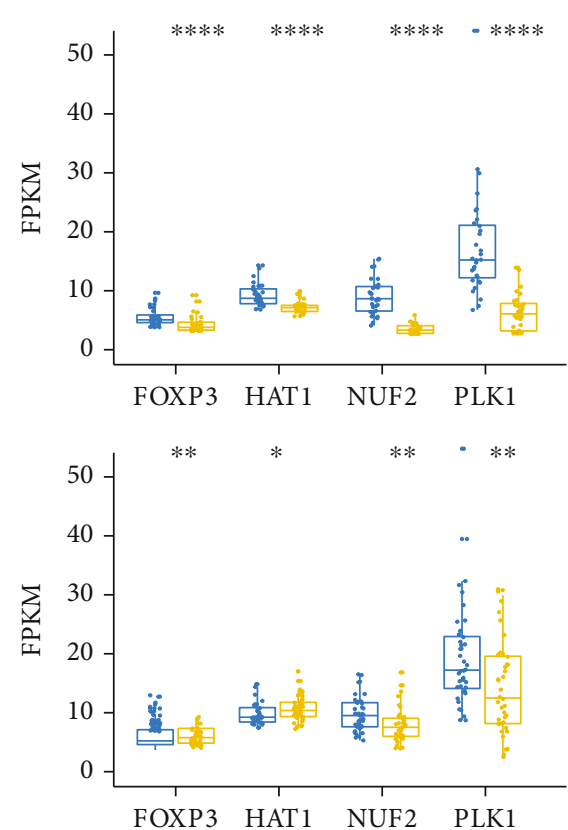

(d)

FIgURE 4: Continued. 


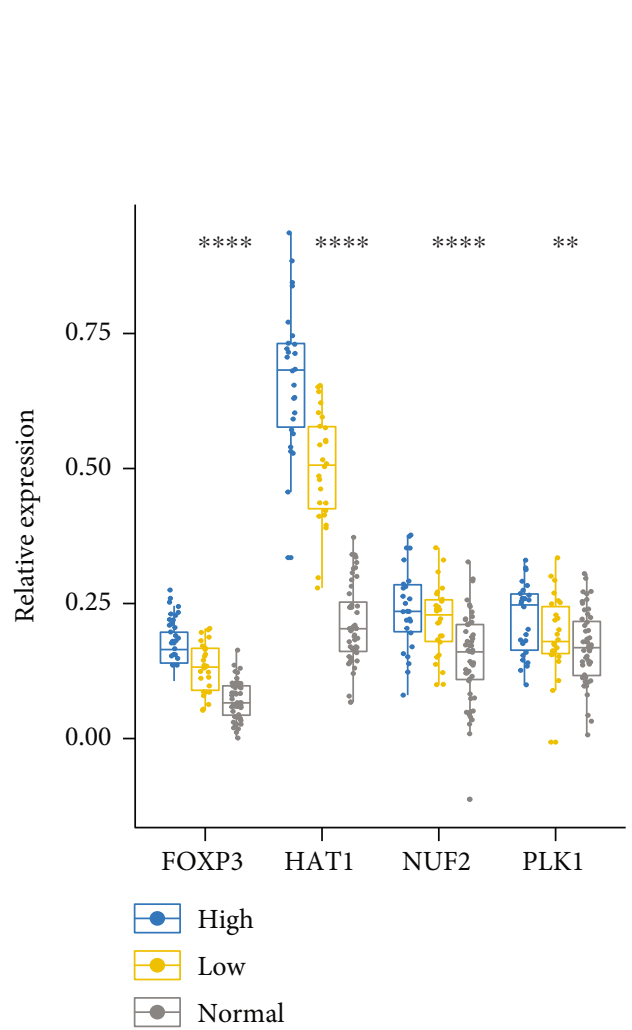

(e)
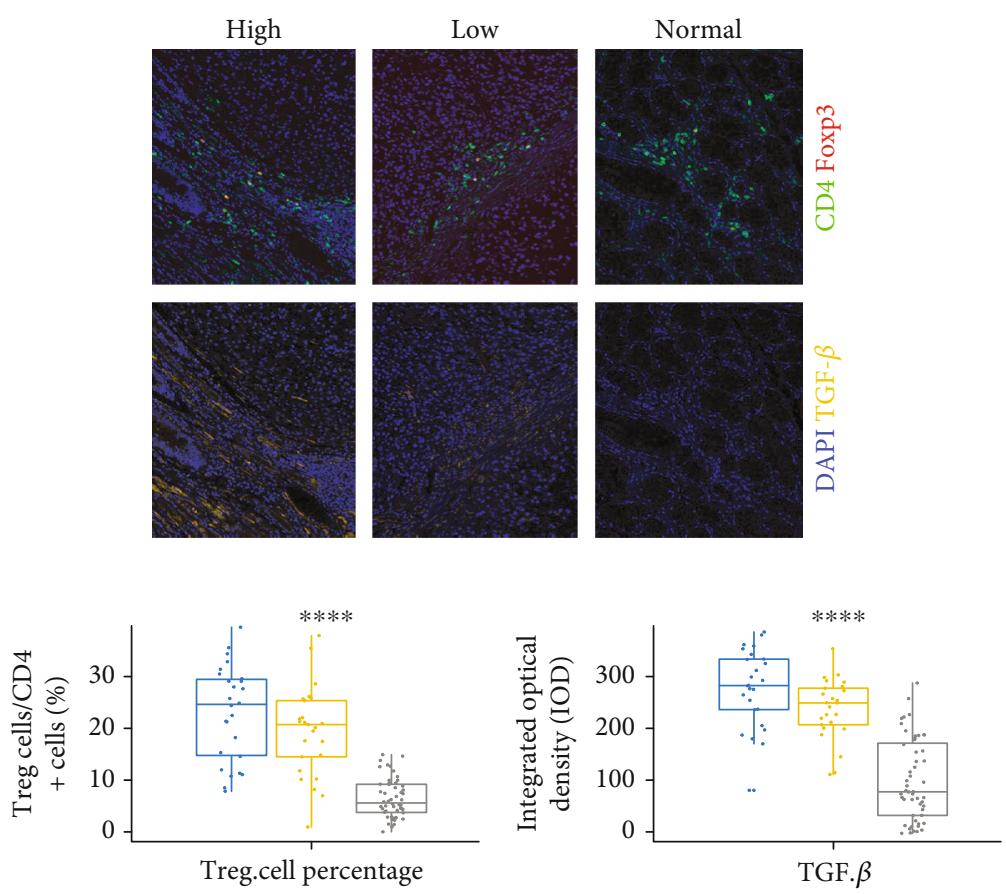

$$
\begin{aligned}
& \text { High } \\
& \text { Low } \\
& \text { Normal }
\end{aligned}
$$

FIGURE 4: RP11-357H14.17 promotes the immunosuppression in human gastric cancer tissues by increasing Treg cell percentage. Heatmap of all significantly different gene sets of c7 ssGSEA analysis for comparison between cancer and normal control samples (a) and RP11357H14.17 high and low expression samples (b) from TCGA-STAD. The "Treg" cell-related gene sets were highlighted in red font. (c) Heatmap of up-regulated genes in "GSE37532_TREG_VS_TCONV_CD4_TCELL_FROM_UP" gene set derived from comparison between cancer and normal control samples and RP11-357H14.17 high and low expression samples from TCGA-STAD, and the representative genes were highlighted in bold. (d) Representative gene expression stands for "GSE37532_TREG_VS_TCONV_CD4_ TCELL_FROM_UP” gene set was compared between cancer and normal control samples, RP11-357H14.17 high and low expression samples from TCGA-STAD, ${ }^{*} p<0.05,{ }^{* *} p<0.01$, and ${ }^{* * * *} p<0.0001$, by unpaired $t$ test, and 52 paired GC tissues categorized as RP11$357 \mathrm{H} 14.17$ high, low expression, and normal control, ${ }^{* * * *} p<0.0001$, one-way ANOVA (e). (f) Representative multiple-color IHC staining figures for CD4, Foxp3, and TGF- $\beta$ protein and comparison between 52 paired GC tissues categorized as RP11-357H14.17 high, low expression, and normal control. ${ }^{* * *} p<0.0001$, one-way ANOVA.

ATF2 activation in RP11-357H14.17 high expression GC tissues might also be related to increased Treg cell percentage.

By using ssGSEA on $c 7$ gene sets, we found the high expression of RP11-357H14.17 was also associated with Treg cell-related immunosuppression, which can also be the other reason for RP11-357H14.17-related tumorigenesis. Regulatory T cells (Tregs) are a specific subset of $\mathrm{T}$ cells whose role is to suppress the immune response, thereby maintaining homeostasis and self-tolerance [21]. Studies have shown that Treg can inhibit $\mathrm{T}$ cell proliferation and cytokine production and play an important role in preventing autoimmunity [21].

In summary, by using bioinformatic analysis, we found that RP11-357H14.17 was overexpressed within human gastric cancer tissues. High expression of RP11-357H14.17 was associated with higher TMN stage, poor prognosis, and poor differentiation by promoting DNA replication. Deeper analysis revealed that RP11-357H14.17 was associated through ATF2 signaling and enhanced immunosuppression by promoting Treg cell percentage within human GC tissues.

\section{Abbreviations}

Gene: Full name

RECQL4: Homo sapiens RecQ protein-like 4

ATAD5: Homo sapiens ATPase family, AAA domain containing 5

GINS1: Homo sapiens GINS complex subunit 1 (Psf1 homolog)

ORC6: Homo sapiens origin recognition complex, subunit 6

RAD51: Homo sapiens RAD51-associated protein 2 (RAD51AP2)

EME1: Homo sapiens essential meiotic endonuclease 1

CCND1: Homo sapiens cyclin D1 (CCND1)

DBF4: Homo sapiens DBF4 homolog B (S. cerevisiae)

MCM2: Homo sapiens minichromosome maintenance complex component 2

NASP: Homo sapiens nuclear autoantigenic sperm protein (histone-binding) 
EZH2: Homo sapiens enhancer of zeste homolog 2 (Drosophila) (EZH2)

PCNA: Homo sapiens proliferating cell nuclear antigen

CDC25A: Homo sapiens cell division cycle 25 homolog A

KRT8: Homo sapiens keratin 85

THOC6: Homo sapiens THO complex 6 homolog (Drosophila)

WT1: Homo sapiens SWT1 RNA endoribonuclease homolog

ATF2: $\quad$ Homo sapiens basic leucine zipper transcription factor, ATF-like 2

FOXP3: Homo sapiens forkhead box P3 (FOXP3)

HAT1: Homo sapiens histone acetyltransferase 1

NUF2: Homo sapiens NUF2, NDC80 kinetochore complex component, homolog

PLK1: Homo sapiens M-phase specific PLK1 interacting protein.

\section{Data Availability}

The RNA-seq data supporting the findings of this study have been deposited in the Cancer Genome Atlas (TCGA) repository (TCGA-STAD). The other data are included within the article.

\section{Conflicts of Interest}

The authors declare that they have no conflicts of interest.

\section{Authors' Contributions}

Tang Xiaoli, Wang Wenting, and Zhang Meixiang contributed equally to this work.

\section{References}

[1] D. H. Ilson, "Advances in the treatment of gastric cancer," Current Opinion in Gastroenterology, vol. 34, no. 6, pp. 465468, 2018.

[2] S. Lauricella, M. Caricato, G. Mascianà et al., "Topographic lymph node staging system shows prognostic superiority compared to the 8th edition of AJCC TNM in gastric cancer. A western monocentric experience," Surgical Oncology, vol. 34, pp. 223-233, 2020.

[3] V. Fort, G. Khelifi, and S. M. I. Hussein, "Long non-coding RNAs and transposable elements: a functional relationship," Biochimica et Biophysica Acta (BBA) - Molecular Cell Research, vol. 1868, no. 1, article 118837, 2021.

[4] Z. Zhao, S. Liang, and F. Sun, "LncRNA DLX6-AS1 promotes malignant phenotype and lymph node metastasis in prostate cancer by inducing LARGE methylation," Frontiers in Oncology, vol. 10, p. 1172, 2020.

[5] M. Zhu, L. Yang, and X. Wang, "NEAT1 knockdown suppresses the cisplatin resistance in ovarian cancer by regulating miR-770-5p/PARP1 axis," Cancer Management and Research, vol. Volume 12, pp. 7277-7289, 2020.

[6] C. Mathias, L. E. Garcia, M. D. Teixeira et al., "Polymorphism of lncRNAs in breast cancer: meta-analysis shows no association with susceptibility," The Journal of Gene Medicine, vol. 22, no. 12, article e3271, 2020.
[7] L. S. Zeng, C. Liu, T. P. Guan et al., "Low expression of lncRNA MGC27345 is associated with poor prognosis in gastric cancer patients," European Review for Medical and Pharmacological Sciences, vol. 24, no. 15, pp. 7982-7990, 2020.

[8] M. Schaalan, W. Mohamed, and S. Fathy, "MiRNA-200c, MiRNA-139 and ln RNA H19; new predictors of treatment response in H-pylori- induced gastric ulcer or progression to gastric cancer," Microbial Pathogenesis, vol. 149, p. 104442, 2020.

[9] P. Qi, M. D. Xu, X. H. Shen et al., "Reciprocal repression between TUSC7 and miR-23b in gastric cancer," International Journal of Cancer, vol. 137, no. 6, pp. 1269-1278, 2015.

[10] J. Jiao and S. Zhang, "Long non-coding RNA MEG-3 suppresses gastric carcinoma cell growth, invasion and migration via EMT regulation," Molecular Medicine Reports, vol. 20, no. 3, pp. 2685-2693, 2019.

[11] Q. Dai, T. Zhang, and C. Li, "LncRNA MALAT1 regulates the cell proliferation and cisplatin resistance in gastric cancer via PI3K/AKT pathway," Cancer Management and Research, vol. Volume 12, pp. 1929-1939, 2020.

[12] Z. Tang, C. Li, B. Kang, G. Gao, C. Li, and Z. Zhang, "GEPIA: a web server for cancer and normal gene expression profiling and interactive analyses," Nucleic Acids Research, vol. 45, no. W1, pp. W98-W102, 2017.

[13] W. Cao, H. Lee, W. Wu et al., "Multi-faceted epigenetic dysregulation of gene expression promotes esophageal squamous cell carcinoma," Nature Communications, vol. 11, no. 1, p. $3675,2020$.

[14] D. Colognori, H. Sunwoo, D. Wang, C. Y. Wang, and J. T. Lee, "Xist repeats A and B account for two distinct phases of $\mathrm{X}$ inactivation establishment," Developmental Cell, vol. 54, no. 1, pp. 21-32.e5, 2020, e5.

[15] B. Yang, T. Luo, M. Zhang, Z. Lu, X. Xue, and G. Fang, "The novel long noncoding RNA RP11-357H14.17 acts as an oncogene by promoting cell proliferation and invasion in diffusetype gastric cancer," Oncotargets and Therapy, vol. Volume 10, pp. 2635-2643, 2017.

[16] L. Gao, X. Nie, W. Zhang et al., "Identification of long noncoding RNA RP11-89K21.1 and RP11-357H14.17 as prognostic signature of endometrial carcinoma via integrated bioinformatics analysis," Cancer Cell International, vol. 20, no. 1, 2020.

[17] G. Watson, Z.'e. A. Ronai, and E. Lau, “ATF2, a paradigm of the multifaceted regulation of transcription factors in biology and disease," Pharmacological Research, vol. 119, pp. 347$357,2017$.

[18] K. Huebner, J. Procházka, A. C. Monteiro, V. Mahadevan, and R. Schneider-Stock, "The activating transcription factor 2: an influencer of cancer progression," Mutagenesis, vol. 34, no. 56, pp. 375-389, 2019.

[19] M. Gozdecka and W. Breitwieser, "The roles of ATF2 (activating transcription factor 2) in tumorigenesis," Biochemical Society Transactions, vol. 40, no. 1, pp. 230-234, 2012.

[20] Y. Liu, Y. Wang, W. Li, P. Zheng, and Y. Liu, “Activating transcription factor 2 and c-Jun-mediated induction of FoxP3 for experimental therapy of mammary tumor in the mouse," Cancer Research, vol. 69, no. 14, pp. 5954-5960, 2009.

[21] D. Wolf, S. Sopper, A. Pircher, G. Gastl, and A. M. Wolf, "Treg(s) in cancer: friends or foe?," Journal of Cellular Physiology, vol. 230, no. 11, pp. 2598-2605, 2015. 